\title{
Linking cellular stress responses to systemic homeostasis
}

Lorenzo Galluzzi 1,2,3,10*, Takahiro Yamazaki' and Guido Kroemer 3 ,4,5,6,7,8,9,10*

Abstract | Mammalian cells respond to stress by activating mechanisms that support cellular functions and hence maintain microenvironmental and organismal homeostasis. Intracellular responses to stress, their regulation and their pathophysiological implications have been extensively studied. However, little is known about the signals that emanate from stressed cells to enable a coordinated adaptive response across tissues, organs and the whole organism. Considerable evidence has now accumulated indicating that the intracellular mechanisms that are activated in response to different stresses - which include the DNA damage response, the unfolded protein response, mitochondrial stress signalling and autophagy - as well as the mechanisms ensuring the proliferative inactivation or elimination of terminally damaged cells - such as cell senescence and regulated cell death - are all coupled with the generation of signals that elicit microenvironmental and/or systemic responses. These signals, which involve changes in the surface of stressed cells and/or the secretion of soluble factors or microvesicles, generally support systemic homeostasis but can also contribute to maladaptation and disease.

\footnotetext{
Pattern recognition receptors

Evolutionarily conserved receptors that elicit inflammation and innate immunity upon recognition of conserved microbial products or endogenous danger signals.

Regulated cell death (RCD). Variant of cell death that relies on a dedicated, genetically encoded machinery and hence can be delayed or accelerated with pharmacological or genetic interventions.
}

One of the reasons behind the evolutionary success of mammals (and other multicellular organisms) is their extraordinary capacity to adapt to changing environmental conditions. In over three decades of cell biology research, we obtained considerable insights into the molecular mechanisms whereby individual cells (attempt to) cope with perturbations of homeostasis. Such mechanisms of cellular adaptation to stress contribute to the formidable resilience of the organism but can also contribute to its degeneration over time, invariably culminating in ageing and/or disease ${ }^{1}$.

Prominent examples of perturbations that induce cell stress include DNA-damaging agents (for example, ionizing radiation and some xenobiotics), which activate repair pathways specific for different types of genetic lesion ${ }^{2}$; heat shock or chemical toxins that cause protein denaturation, both of which activate the unfolded protein response (UPR) in the endoplasmic reticulum (ER) and mitochondria ${ }^{3,4}$; hypoxia, respiratory poisons and xenobiotics that cause mitochondrial stress ${ }^{5}$; nutrient deprivation, which activates autophagy in most cells of the organisms, hence enabling them to catabolize their own components for survival ${ }^{6,7}$; and infectious agents, which can drive a plethora of stress responses by activating pattern recognition receptors ${ }^{8}$. The triggers, receptors, signal transducers, effector mechanisms and adaptive responses that operate at the cellular level in each of these scenarios have been characterized in great molecular detail. As a common theme, it appears that perturbations of cellular homeostasis either lead to management of stress and damage repair - generally along with transient alterations in cellular metabolism or culminate in cellular senescence or regulated cell death (RCD) when the restoration of normal cellular functions is impossible $e^{9,10}$. The latter scenarios may be viewed as mechanisms for the preservation of organismal homeostasis, as severely damaged, irreversibly infected, functionless and/or potentially oncogenic cells are destined for persistent inactivation or elimination, respectively ${ }^{11}$.

It has become apparent that most (if not all) mechanisms of cellular response to stress are also associated with paracrine and endocrine signals that communicate a potential threat to the organism and hence contribute to the maintenance of systemic homeostasis ${ }^{1,12-14}$ (FIG. 1). This intercellular communication is achieved via multiple mechanisms, including (but not limited to) alterations in the shape of stressed cells and their connections with the microenvironment; the exposure of specific molecules on their surface; and the active or passive release of bioactive factors such as ions, small metabolites, intracellular proteins, cytokines or microvesicles.

Here, we review how distinct stress responses are relayed from the intracellular to the extracellular milieu, how such communication favours the maintenance of organismal homeostasis and how deviations from its normal course may drive maladaptation and disease. kroemer@orange.fr

https://doi.org/10.1038/ s41580-018-0068-0 
Cell cycle checkpoints

Control mechanisms that ensure the progression of eukaryotic cells along the cell cycle only in the presence of favourable conditions.

Immunogenicity

The ability to trigger an immune response, resulting

from antigenicity (the property of being recognized by immune cells) and adjuvanticity (the property of delivering activating signals to immune cells).

Natural killer (NK) cells

A group of cells from the innate lymphoid immune system that can mediate cytotoxic functions independent of antigen recognition

Major histocompatibility complex

(MHC). Set of cell surface proteins essential for the immune system to recognize foreign molecules in vertebrates.

Abscopal responses Measurable reductions in the size of a non-irradiated tumour or metastasis thereof following the irradiation of another lesion.

Hypofractionated irradiation

The delivery of radiation therapy in a few fractions, each with a larger dose than the standard 1.8 or $2 \mathrm{~Gy}$.

Micronuclei

Small enveloped structures that encompass chromosomes of fragments thereof that are not incorporated into one of the daughter nuclei during mitosis.

Chromothripsis

A process whereby up to thousands of clustered chromosomal rearrangements occur in a single event in localized genomic regions affecting one or a few chromosomes.

\section{Adaptation to cellular stress}

Multiple potentially detrimental perturbations of the intracellular or extracellular microenvironment can be successfully managed by mammalian cells upon the activation of stress responses that preserve cellular functions and repair macromolecular damage.

The DNA damage response. DNA damage leads to the activation of cell cycle checkpoints coupled to the recruitment of the DNA repair machinery to genetic lesions. If the DNA damage response (DDR) is unsuccessful, the same machinery ultimately initiates cellular senescence or $\mathrm{RCD}^{2}$ (BOX 1), both of which also impinge on the control of microenvironmental and systemic homeostasis (see separate sections below). Importantly, the DDR itself is also intimately connected with the elimination of damaged cells by immune effectors and the establishment of inflammatory responses that contribute to the maintenance of local and systemic homeostasis ${ }^{15}$. In some cases, however, such responses can favour maladaptation and contribute to the establishment of pathological conditions, especially in the presence of molecular defects in the mechanisms that physiologically extinguish inflammation ${ }^{16}$.

DNA-damaging agents including ionizing radiation are known for their ability to change the immunogenicity of affected cells, hence facilitating their recognition by cytotoxic T lymphocytes (CTLs) or natural killer (NK) cells. Activation of the DDR has been linked to increased expression of major histocompatibility complex (MHC) class I molecules on the cell surface, resulting in increased susceptibility to recognition by CTLs. This increased recognition is particularly important for the elimination of malignant cells that express antigens potentially detectable by the immune system ${ }^{17}$. Moreover, DNA damage generally favours the upregulation of multiple ligands for NK cell activatory receptors, such as NKG2D type II integral membrane protein also known as KLRK1) and DNAX accessory molecule 1 (DNAM1; also known as CD226 (REFS $\left.{ }^{18,19}\right)$ ). The increased expression of these molecules is particularly relevant when genetic integrity cannot be restored, resulting in cell senescence $^{20}$. In this context, NK cells remove cells with unrepairable DNA defects that would otherwise

\footnotetext{
Author addresses

${ }^{1}$ Department of Radiation Oncology, Weill Cornell Medical College, New York, NY, USA.

${ }^{2}$ Sandra and Edward Meyer Cancer Center, New York, NY, USA.

${ }^{3}$ Université Paris Descartes/Paris V, Paris, France. ${ }^{4}$ Université Pierre et Marie Curie/Paris VI, Paris, France. ${ }^{5}$ Equipe 11 Labellisée Ligue contre le Cancer, Centre de Recherche des Cordeliers, Paris, France. 'INSERM, U1138, Paris, France.

${ }^{7}$ Metabolomics and Cell Biology Platforms, Gustave Roussy Comprehensive Cancer Institute, Villejuif, France.

${ }^{8}$ Department of Women's and Children's Health, Karolinska University Hospital, Stockholm, Sweden.

'Pôle de Biologie, Hopitâl Européen George Pompidou, AP-HP, Paris, France.

${ }^{10}$ These authors share senior authorship: Lorenzo Galluzzi, Guido Kroemer.
}

persist (although in a senescent, non-proliferative state) and potentially contribute to tissue deterioration and ageing (see below). Furthermore, accumulating evidence suggests that the DDR-mediated activation of NK cells contributes to the recognition and removal of pre-malignant and malignant cells and supports oncosuppression $^{21,22}$. Of note, NK cell-activating ligands can be shed by metalloproteinases secreted in the tumour microenvironment, resulting in the generation of decoy molecules that inhibit (rather than activate) NK cells ${ }^{21}$.

DNA damage also initiates the secretion of soluble factors that regulate local inflammatory responses, both in the context of cellular senescence and independently of it. One of the mechanisms underlying this process involves the DNA damage-associated release of nuclear DNA fragments into the cytoplasm ${ }^{23-26}$. Cytosolic DNA is recognized by cyclic GMP-AMP synthase (cGAS), which drives the activation of stimulator of interferon genes (STING; encoded by TMEM173) and the consequent production of type I interferon (IFN) ${ }^{27,28}$. Because type I IFN is a strongly pro-inflammatory cytokine, its production needs to be tightly regulated to avoid tissue damage related to uncontrolled inflammation. Type I IFN secretion driven by cytosolic DNA recognition is negatively controlled by cytosolic nucleases, including three-prime repair exonuclease 1 (TREX1) and RNase H2 (REFS ${ }^{29,30}$ ) (FIG. 2a). Accordingly, lossof-function mutations in TREX1 or any of the three subunits of RNase $\mathrm{H} 2$ (that is, RNASEH2A, RNASEH2B and RNASEH2C) have been associated with AicardiGoutières syndrome, an autoimmune disorder characterized by deregulated type I IFN production ${ }^{27}$. Of note, excessive cGAS-STING-dependent type I IFN secretion has also been involved in pathological maladaptation following myocardial infarction ${ }^{31}$. These observations exemplify how the deregulated release of type I IFN can contribute to disease.

That being said, production of type I IFN is critical for cancer immunosurveillance as it boosts the activity of multiple immune effector cells. To counteract this effect, cancer cells can upregulate TREX1 in response to DNA damage. Notably, high-dose irradiation - which potently induces TREX1 expression - is unable to drive type I IFN-dependent abscopal responses in mice, in contrast to hypofractionated irradiation, which does not induce TREX1 expression $^{25}$. These observations can explain, at least in part, why hypofractionated irradiation exerts superior immunostimulatory effects and enhanced tumour control as compared with single high-dose radiation therapy in multiple preclinical settings ${ }^{32,33}$.

Ionizing irradiation and drugs that interfere with mitosis (such as the microtubular poison nocodazole) can also generate chromosome fragments or promote the missegregation of entire chromosomes, hence favouring the formation of micronuclei ${ }^{23,26}$. A frequent event in micronuclei, the formation of which is favoured in the presence of RNase $\mathrm{H} 2$ defects $^{34}$, is chromothripsis ${ }^{35}$. Chromothripsis is advantageous to progressing tumours as a source of clonal diversity (and hence cellular fitness) ${ }^{36}$. However, chromothripsis is also associated with the breakdown of the micronuclear envelope, 


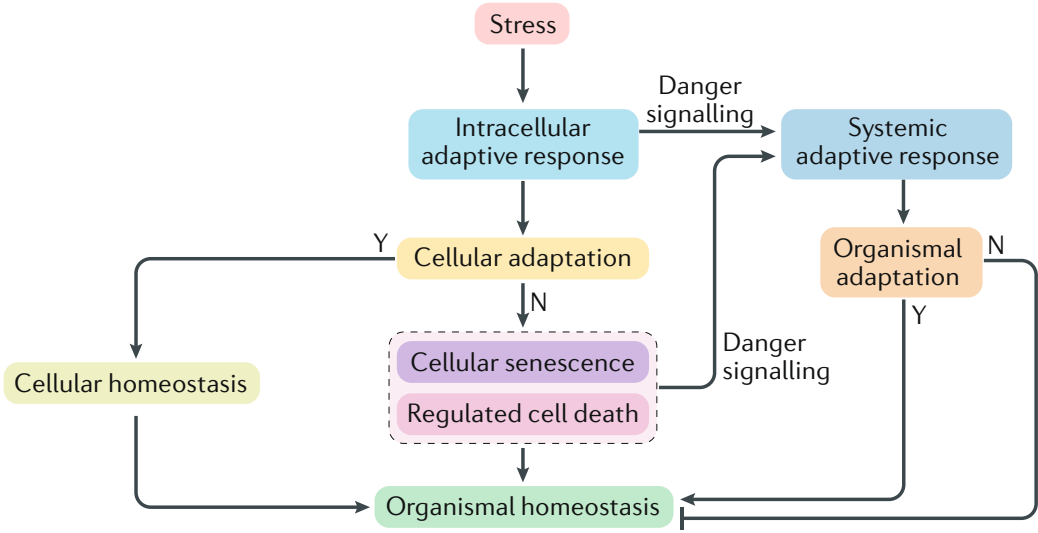

Fig. 1 | Integration of cellular and systemic stress responses and their roles in the maintenance of organismal homeostasis. Mammalian cells respond to perturbations of the intracellular or extracellular microenvironment with mechanisms that are aimed at restoring cellular homeostasis. The successful preservation of physiological cellular functions supports, by extension, the maintenance of organismal homeostasis. If microenvironmental fluctuations are too intense or prolonged for cellular adaptation, however, mammalian cells actively undergo either senescence, resulting in their permanent proliferative inactivation, or regulated cell death (RCD), resulting in their elimination. Both cellular senescence and RCD constitute mechanisms for the preservation of systemic homeostasis when physiological cellular functions are terminally lost. Moreover, cellular adaptation to stress (be it successful, leading to damage repair and recovery, or not successful, leading to proliferative inactivation or elimination) is intimately linked to cell-extrinsic mechanisms that operate locally or systemically in support of organismal homeostasis. Thus, cellular responses to potentially threatening perturbations are efficiently relayed to the local and systemic microenvironment via a large panel of danger signals that operate as paracrine or endocrine mediators to promote the maintenance of organismal fitness.

Proteostasis

The maintenance of protein homeostasis within a defined organelle, cell or tissue, which involves correct protein synthesis, folding, distribution and degradation.

Myokine

One of several small proteins or proteoglycans that are released by myocytes upon contraction to mediate autocrine, paracrine or endocrine effects

Nuclear factor- $\kappa \mathrm{B}(\mathrm{NF}-\kappa \mathrm{B})$ signalling

Biological output of NF-кBdependent transcription, generally involving a robust pro-inflammatory component.

Hepcidin antimicrobial peptide

(HAMP). Key negative regulator of circulating iron availability in mammals, promoting a state of accrued bacterial resistance. which exposes DNA to the cytoplasm, followed by cGAS-STING activation, type I IFN secretion and consequent establishment of systemic immunity and immunosurveillance ${ }^{23,26}$. Accordingly, the therapeutic effect of microtubule-targeting anticancer agents (such as taxanes) might be, at least in part, linked to their ability to provoke micronucleation and consequent type I IFN secretion ${ }^{37}$, whereas the clinical failure of other antimitotic drugs may reflect their inability to trigger this process ${ }^{38}$. Thus, cancer cells may benefit from the inactivation of cGAS, STING or their downstream effectors, including interferon regulatory factor 3 (IRF3) ${ }^{39}$. Of note, autophagy efficiently removes micronuclei ${ }^{40-42}$ and hence might have an ambiguous role in tumour progression by avoiding chromothripsis (which would reduce the pace of clonal evolution) but at the same time suppressing micronuclei-driven type I IFN signalling (which would compromise immunosurveillance). Whether the autophagic degradation of micronuclei contributes to the well-established dual role of autophagy in tumour progression ${ }^{43,44}$ remains unresolved.

Additional links between the DDR and systemic homeostasis have been elucidated in Caenorhabditis elegans. DNA damage in C. elegans germ cells drives the activation of MPK1, the worm orthologue of mammalian MAPK3 and MAPK1 (REFS ${ }^{45}$ ), which causes the release of peptides that promote innate immunity response, followed by the activation of the ubiquitin-proteasome system in somatic tissues, enhanced proteostasis and systemic stress resistance ${ }^{45}$. In another study, localized exposure of nematodes to ultraviolet light to induce DNA damage was shown to trigger a systemic stress response that was dependent on cep-1, the orthologue of mammalian TP53 (encoding p53), and on cpr-4, the orthologue of mammalian CTSB (encoding cathepsin B). In this case, local activation of CEP-1 promoted expression and secretion of CPR-4 into the extracellular fluids and into the culture medium. CPR-4 release was associated with reduced cell death in the non-irradiated tissues and neighbouring animals, as well as with larval lethality. Subsequently, CPR- 4 was mechanistically identified as the factor responsible for these radiation-induced bystander effects $^{46}$. This mechanism is an interesting example of intracellular adaptation to stress relaying a signal not only to the entire organism but also to other individuals. It remains to be seen whether extracellular CTSB (which is normally confined within lysosomes) also participates in the bystander effects of radiation in mammals. Of note, extracellular CTSB has been documented in human neoplasm $\mathrm{s}^{47}$ and may influence tumour progression as well as responses to treatment ${ }^{48,49}$. Intriguingly, in mice, monkeys and humans, circulating CTSB levels are increased in response to exercise. In this context, CTSB was shown to act as a myokine that is required for the running-stimulated increase in adult hippocampal neurogenesis and spatial memory function ${ }^{50}$. Thus, CTSB may have multiple paracrine and endocrine effects in mammals as well. Whether CTSB secretion in mammals involves the DDR remains an open question.

Altogether, these examples illustrate some of the mechanisms through which the DDR can initiate paracrine and endocrine signals with adaptive (or maladaptive) consequences. To what degree the type and the extent of DNA damage influence these processes remains to be clarified. Moreover, it remains unclear whether there is a minimal degree of damage that is required to raise the systemic response and to what extent the intracellular output of the DDR (DNA repair) and its extracellular effects (paracrine and endocrine signalling) are coupled.

The unfolded protein response. The UPR operating at the $\mathrm{ER}$, also known as $\mathrm{UPR}^{\mathrm{ER}}$, is an adaptive response aimed at resolving the accumulation of unfolded polypeptides in the ER lumen or eliminating cells that cannot recover reticular proteostasis by inducing $\mathrm{RCD}^{3}$ (BOX 2). In conjunction, the $U P R^{\mathrm{ER}}$ is able to initiate inflammatory responses that - if unresolved contribute to pathogenic maladaptation, metabolic disorders and accelerated ageing ${ }^{51}$. For instance, renal epithelial cells subjected to ER stress secrete angiogenin (ANG) as a consequence of the activation of inositolrequiring enzyme $1 \alpha$ (IRE1 $\alpha$; encoded by ERN1) and nuclear factor- $\mathrm{KB}$ (NF-KB) signalling, which favours the establishment of inflammation by tissue-resident macrophages $^{52}$. Similarly, hepatocytes responding to ER stress secrete hepcidin antimicrobial peptide (HAMP) following the $\mathrm{UPR}^{\mathrm{ER}}$-dependent activation of cAMPresponsive element-binding protein 3 -like protein 3 $\left(\right.$ CREB3L3) ${ }^{53}$. Circulating HAMP ultimately causes hypoferraemia and splenic iron sequestration in mice ${ }^{53}$. This mechanism links the $\mathrm{UPR}^{\mathrm{ER}}$ in a specific 
Brown adipose tissue

Highly specialized adipose tissue, the main function of which is to produce heat (thermogenesis) compartment (the liver) with organismal iron homeostasis, specifically with a reduction in systemic iron that mediates antibacterial effects (FIG. 2b).

Induction of RCD by the $\mathrm{UPR}^{\mathrm{ER}}$ is mainly driven by deregulated protein synthesis, which results from the activation of C/EBP-homologous protein (CHOP; also known as DDIT3). CHOP is a target of activating transcription factor 4 (ATF4; one of the main $\mathrm{UPR}^{\mathrm{ER}}$ effectors; see BOX 2) and transactivates several genes involved in translation ${ }^{3}$. In cells that are susceptible to ER stress, such as intestinal epithelial cells (IECs), CHOP also transactivates genes coding for NKG2D ligands such as UL16-binding protein 1 (ULBP1), potentially driving a spontaneous intestinal inflammation (enteritis) consequent on local NK cell activation ${ }^{54}$. Thus, the $\mathrm{UPR}^{\mathrm{ER}}$ resembles the DDR in its ability to promote the restoration of cellular homeostasis or, when this is not possible, to drive RCD along with the activation of local inflammation and innate immunity.

Interestingly, ectopic activation of ATF4 in the skeletal muscle by means of a ligand-activated variant of PKR-like ER kinase (PERK; also known as EIF2AK3; see $B O X 2$ ) expressed under the control of a tissue-specific promoter drives the secretion of fibroblast growth factor 21 (FGF21), an anti-obesity myokine ${ }^{55}$. This finding may explain how muscle-specific PERK activation stimulates energy consumption by the brown adipose tissue,

\section{Box 1 | The DNA damage response at a glance}

Nuclear DNA can be damaged by external cues (for example, ultraviolet light and xenobiotics) and by endogenous causes, including errors in DNA replication or DNA maintenance. The term DNA damage response (DDR) generally refers to a highly interconnected network of molecular pathways that is in place to sense and manage specific genetic lesions as they form. Similar to most cellular mechanisms of adaptation to stress, the DDR can enable tolerance of damage, repair or the permanent inactivation (via cellular senescence or regulated cell death (RCD)) of cells bearing non-tolerable or non-repairable lesions.

There are multiple pathways whereby the DDR initially attempts to repair DNA damage, including (but not limited to) base excision repair (BER), which operates on DNA lesions that do not impose large distortions to the DNA double helix; nucleotide excision repair (NER), which corrects a broad panel of DNA lesions, including adducts and other bulky structures that distort the double helix; DNA mismatch repair (MMR), which resolves base mismatches as well as small insertions or deletions; nonhomologous end-joining (NHEJ), which is the main pathway whereby mammalian cells fix double-strand breaks (DSBs); and homologous recombination (HR), which also repairs DSBs but is active only in the $\mathrm{S}$ and $\mathrm{G} 2$ phases of the cell cycle ${ }^{180}$. Moreover, socalled translesion synthesis (TLS) bypasses, but does not remove, DNA lesions that stall the replication machinery and would otherwise cause its collapse or introduce DSBs. Of note, TLS is a major mechanism of tolerance to DNA damage and is generally associated with an increased mutational rate as compared with conventional DNA replication.

Two major players in the DDR of mammalian cells are ataxia telangiectasia mutated (ATM) and ataxia telangiectasia and Rad3-related protein (ATR). Upon recruitment to damaged DNA via dedicated supramolecular platforms, ATM and ATR phosphorylate, hence activating, a wide panel of factors involved in the preservation of nuclear and extranuclear homeostasis, including histone $\mathrm{H} 2 \mathrm{AX}$, checkpoint kinase 2 (CHEK2) and CHEK1, which culminates with the stabilization of tumour suppressor p53. Initially, p53 supports repair mechanisms by transactivating genes that enable a reversible cell cycle arrest and maintain metabolic homeostasis. However, if genetic lesions cannot be resolved, p53 acquires the ability to efficiently drive cellular senescence or RCD via transcriptional and transcription-independent mechanisms ${ }^{180}$. Thus, $\mathrm{p} 53$ stands at a crucial position in the molecular mechanisms that determine whether DNA lesions can be repaired (coupled to the restoration of cellular homeostasis) or not (coupled to the proliferative inactivation or elimination of damaged cells in support of organismal homeostasis). hence limiting weight gain in mice receiving a high-fat $\operatorname{diet}^{55}$. Interestingly, the PERK-ATF4-FGF21 axis may also be activated by chronic exercise and exposure to cold temperatures, two conditions that promote $\mathrm{UPR}^{\mathrm{ER}}$ in the skeletal muscle ${ }^{55,56}$. This process exemplifies the endocrine pathways whereby the skeletal muscle affects systemic metabolism. It will be interesting to see whether myokine signalling can be targeted for the treatment of metabolic disorders in humans.

Multiple tissues from obese individuals, including the liver, abdominal fat and hypothalamus, show signs of ER stress, including the phosphorylation of PERK and another $\mathrm{UPR}^{\mathrm{ER}}$ effector, eukaryotic translation initiation factor 2 subunit- $\alpha$ (eIF2 $\alpha$; also known as EIF2S1; see BOX 2) $)^{57}$. There is now evidence that such an adiposity-associated $\mathrm{UPR}^{\mathrm{ER}}$ could potentially contribute to alleviating pathogenesis of the disease. For example, conditional deletion of the $\mathrm{UPR}^{\mathrm{ER}}$ effector Xbpl (see also BOX 2) from neurons and glia increases the susceptibility of mice to diet-induced obesity as it causes severe resistance to the satiety hormone leptin ${ }^{57}$. Conversely, overexpression of spliced XBP1 (XBP1s) in specific hypothalamic neurons contributes to improved hepatic insulin sensitivity and suppression of endogenous glucose production in mice on a high-fat $\operatorname{diet}^{58}$. These metabolic effects are mediated by a brainto-liver communication and are associated with $\mathrm{UPR}^{\mathrm{ER}}$ activation in hepatocytes, which contributes to metabolic health by triggering a transcriptional programme that mimics the postprandial status ${ }^{58}$. The precise mediators of this endocrine propagation of the $\mathrm{UPR}^{\mathrm{ER}}$ remain unknown. Intriguingly, in C. elegans, neuronal expression of XBP1s extends lifespan by stimulating the $\mathrm{UPR}^{\mathrm{ER}}$ in intestinal cells. This pathway involves the release of neurosynaptic vesicles from neurons, suggesting that in this setting the mediator of endocrine UPR ${ }^{\mathrm{ER}}$ induction is a neurotransmitter ${ }^{59}$. It has been speculated that the programme elicited by neuronal XBP1s may deliver pre-emptive signals to peripheral tissues to stimulate a systemic stress response preparing the organism for coping with stress ${ }^{51}$. However, this hypothesis remains to be experimentally validated.

Of note, in some instances (for example, in ageing mammalian cells) the ability of the UPR ${ }^{\mathrm{ER}}$ machinery to efficiently resolve ER stress or drive RCD is limited, resulting in the establishment of chronic ER stress, which is a major determinant of immunological, neurological and metabolic maladaptation ${ }^{51}$. Thus, although the acute $\mathrm{UPR}^{\mathrm{ER}}$ generally supports microenvironmental and systemic homeostasis by favouring cellular adaptation to ER stress or RCD, its chronic counterpart has detrimental effects for the organism ${ }^{51,60,61}$.

Activation of the UPR ${ }^{\mathrm{ER}}$ is also prominent in cancer cells as a mechanism to cope with intense protein synthesis associated with high proliferation rates and in response to microenvironmental inducers of ER stress (for example, some chemotherapeutics, see below) ${ }^{3}$. Importantly, the UPR ${ }^{\mathrm{ER}}$ can be relayed from malignant cells to myeloid cells by thus-far unidentified soluble factors, resulting in the secretion of multiple proinflammatory cytokines, including interleukin 6 (IL-6) and tumour necrosis factor (TNF) ${ }^{62}$. Such a transmissible ER stress (TERS) also operates between cancer cells, leading to the exposure of the ER chaperone BiP (also known 

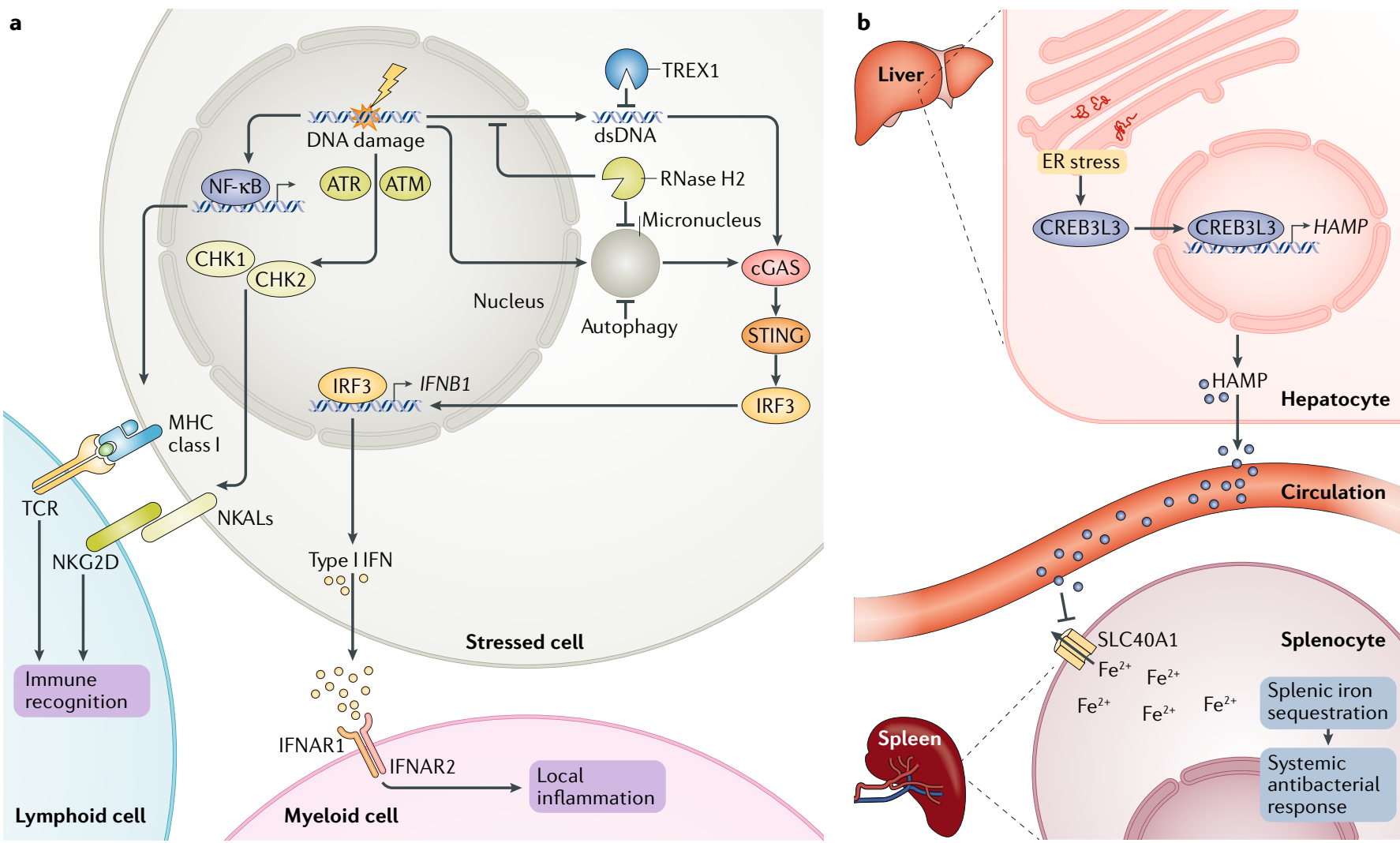

Fig. 2 | The DDR and UPR ${ }^{E R}$ in the regulation of microenvironmental and systemic homeostasis. a | DNA damage in mammalian cells can cause the activation of a transcriptional response driven by nuclear factor- $\mathrm{KB}(\mathrm{NF}-\kappa \mathrm{B})$, culminating with the exposure of major histocompatibility complex (MHC) class I molecules on the cell surface. In conjunction, the detection of genetic lesions by ataxia telangiectasia mutated (ATM) and ataxia telangiectasia and Rad3-related protein (ATR) can stimulate the exposure of natural killer (NK) cell-activating ligands (NKALs) on the outer leaflet of the plasma membrane. Both of these processes facilitate the recognition of cells experiencing DNA damage by lymphoid cells. Moreover, multiple DNA-damaging agents cause the release of double-stranded DNA (dsDNA) into the cytosol or the formation of micronuclei, ultimately leading to type I interferon (IFN) secretion mediated by the activation of cyclic GMP-AMP synthase (cGAS) and stimulator of interferon genes (STING). Upon binding to heterodimeric IFN $\alpha / \beta$ receptors (IFNARs) on myeloid cells, type I IFN stimulates local inflammation. b| Activation of the unfolded protein response (UPR) operating at the endoplasmic reticulum (ER), also known as the UPR ${ }^{E R}$, in hepatocytes has been linked to the transactivation of hepcidin antimicrobial peptide (HAMP) following cAMP-responsive element-binding protein 3-like 3 (CREB3L3) activation. The consequent increase in circulating HAMP enables the sequestration of iron in the spleen upon the HAMP-dependent inhibition of solute carrier family 40 member 1 (SLC40A1) on splenocytes. This reduction in iron availability contributes to the systemic antibacterial response, as bacteria rely on iron uptake for growth. CHK, checkpoint kinase; DDR, DNA damage response; IRF3, interferon regulatory factor 3; NKG2D, NKG2D type II integral membrane protein; TCR, T cell receptor; TREX1, three-prime repair exonuclease 1.

Dendritic cells

Myeloid cells that play a major role in the initiation of T celldependent immune responses.

Myeloid-derived suppressor cells

(MDSCs). A heterogeneous population of cells that are defined by their myeloid origin, immature state and ability to potently suppress T cell responses. as HSPA5 and GRP78) on the cell surface, an IRE1adependent increase in WNT signalling and the activation of the PERK-ATF4 axis, which collectively provide cytoprotection and promote cancer growth. Accordingly, TERS-primed cancer cells grow more quickly in vivo than their control counterparts ${ }^{62}$. Some cell-deathpromoting chemotherapeutic agents such as anthracyclines and oxaliplatin also trigger a partial ER stress response in cancer cells, which manifests with PERKdependent eIF2 $\alpha$ phosphorylation but not with ATF4, ATF6 and XBP1s activation ${ }^{63}$. Phosphorylated eIF2a favours the translocation of ER chaperones including calreticulin (CALR) and protein disulfide-isomerase A3 (PDIA3) from the ER lumen to the cell surface ${ }^{64}$, where they act as 'eat-me' signals to promote phagocytosis of dying cancer cells by dendritic cells and hence initiate anticancer immunity ${ }^{17}$. The clinical relevance of this process is supported by the fact that CALR exposure on cancer cells correlates not only with eIF2a phosphorylation but also with prognostically favourable anticancer immune responses ${ }^{65,66}$. Of note, dying cancer cells can also release soluble CALR, which limits the ability of macrophages to present phagocytosed antigens (as a consequence of MHC class II downregulation) ${ }^{67}$ and favours the accumulation of myeloidderived suppressor cells (MDSCs) ${ }^{68}$, hence potentially promoting tumour progression. Thus, the net microenvironmental effect of $\mathrm{UPR}^{\mathrm{ER}}$-driven CALR signalling may depend on the balance between the surface-bound and soluble variants of the molecule. This finding is reminiscent of the DDR-driven exposure of NK-cell-activating ligands on the surface of cancer cells, which upon 


\section{Box 2 | The UPR ${ }^{E R}$ at a glance}

The unfolded protein response (UPR) operating at the endoplasmic reticulum (ER), also known as the UPR ${ }^{E R}$, is an evolutionarily conserved mechanism for the preservation of proteostasis in the ER under conditions that favour the accumulation of unfolded proteins (for example, impaired $\mathrm{Ca}^{2+}$ homeostasis and viral infection) ${ }^{3,51}$.

In mammalian cells, the acute phase of the UPR ${ }^{E R}$ involves three different signalling modules: the activation of PKR-like ER kinase (PERK) and consequent phosphorylation of eukaryotic translation initiation factor 2 subunit- $\alpha$ (elF2 $\alpha$ ), ultimately resulting in a global rearrangement of translation that limits the synthesis of the majority of proteins but enables expression of activating transcription factor 4 (ATF4); the activation of ATF6 and its translocation from the ER to the nucleus via the Golgi apparatus; and the activation of inositol-requiring enzyme $1 \alpha($ IRE $1 \alpha)$ and consequent alternative splicing of X-box binding protein 1 (XBP1) to generate the active variant of the protein, spliced XBP1 (XBP1s) ${ }^{3,51}$. ATF4, ATF6 and XBP1s act as transcription factors that initially control the expression of genes involved in the mechanisms of homeostatic adaptation to protein overload, including an increased capacity for protein folding and chaperoning within the ER and an increased flux of protein degradation via the proteasome and autophagy ${ }^{3,51}$. One of the main regulators of the UPR ${ }^{E R}$ is an ER chaperone BiP (also known as HSPA5 and GRP78) ${ }^{3,51}$, which inhibits PERK, ATF6 and IRE1 $\alpha$ activation in physiological conditions. Unfolded proteins accumulating in the ER lumen compete for BiP binding with PERK, ATF6 and IRE1 $\alpha$, ultimately leading to UPR ${ }^{\mathrm{ER}}$ activation. Of note, the gene encoding BiP, HSPA5, is transactivated by ATF4, ATF6 and (less so) $\mathrm{XBP} 1 \mathrm{~s}^{181-183}$, and the HSPA5 mRNA can be normally translated even in the context of elF2 $\alpha$ phosphorylation owing to an internal ribosome entry site ${ }^{184}$. Thus, BiP operates within a negative feedback circuitry that attempts to shut down the UPR ${ }^{E R}$ upon restoration of homeostatic conditions s.51. $^{3,5}$.

If reticular proteostasis cannot be recovered, however, prolonged UPR ${ }^{\mathrm{ER}}$ signalling triggers (generally apoptotic) regulated cell death (RCD) following the ATF4-driven activation of $\mathrm{C} / \mathrm{EBP}-$ homologous protein (CHOP). Among various target genes, $\mathrm{CHOP}$ transactivates protein phosphatase 1 regulatory subunit 15A (PPP1R15A), which, in complex with serine/threonine-protein phosphatase PP1a catalytic subunit (PP1A), catalyses the dephosphorylation of elF $2 \alpha$, resulting in high protein synthesis despite persistent protein folding overload ${ }^{3,185}$. In addition to augmenting ER stress, this enables the translation of multiple mRNAs upregulated by $\mathrm{CHOP}$ that code for proapoptotic factors, including those that encode BCL-2 binding component 3 (BBC3), BCL-2-interacting mediator of cell death (BIM; encoded by BCL2L11) and tumour necrosis factor receptor superfamily member 10B (TNFRSF10B) ${ }^{186,187}$. Thus, at the cell-intrinsic level, the acute phase of the UPR ${ }^{\mathrm{ER}}$ operates as a conventional adaptive response to stress as it initially attempts to restore cellular homeostasis but elicits RCD if cellular functions are irremediably compromised ${ }^{3,51}$.

Integrated stress response Evolutionarily conserved homeostatic programme common to all eukaryotic cells.

Mitokines

Soluble factors released by cells experiencing mitochondrial stress and operating as autocrine, paracrine or endocrine mediators. solubilization by metalloproteinases inhibit cancer recognition by NK cells (see above). Overall, although the $\mathrm{UPR}^{\mathrm{ER}}$ in living malignant cells mostly promotes cancer growth by cell-intrinsic and cell-extrinsic mechanisms, the UPR ${ }^{\mathrm{ER}}$ coupled to cancer cell death generally stimulates anticancer immunity.

In summary, there are multiple pathways whereby the $U P R^{\mathrm{ER}}$ is relayed to other cells and tissues, many of which occur in the context of human disease. Interestingly, although the cellular output of the $\mathrm{UPR}^{\mathrm{ER}}$ (recovery of ER homeostasis or RCD activation) almost invariably involves the three arms of the process (BOX 2), the same does not always apply to $U R^{\mathrm{ER}}$-driven paracrine and endocrine signalling. Therefore, it is tempting to speculate that one or more branches of the $\mathrm{UPR}^{\mathrm{ER}}$ may have evolved specifically to link intracellular response to stress with organismal homeostasis. This possibility remains to be experimentally investigated.

Mitochondrial stress signalling. The accumulation of unfolded proteins in the mitochondrial matrix drives a variant of the UPR (commonly referred to as $U P R^{\mathrm{mt}}$ ), which resembles the $\mathrm{UPR}^{\mathrm{ER}}$ in its general organization - namely, the activation of a broad transcriptional response aimed at the restoration of mitochondrial proteostasis ${ }^{69-71}$. Although they share some signal transducers and effectors (see below), the UPR ${ }^{\mathrm{mt}}$ and the UPR ${ }^{\mathrm{ER}}$ should be considered as two distinct processes, not only because they respond to stress at two different cellular compartments but also because they have cellular, local and systemic consequences that only partially overlap ${ }^{51,72}$. Of note, $\mathrm{UPR}^{\mathrm{mt}}$ is an evolutionarily conserved process, and UPR ${ }^{\mathrm{mt}}$-like responses have been documented in multiple eukaryotes other than mammals, including plants, flies and nematodes ${ }^{72-74}$.

In C. elegans, the UPR ${ }^{\mathrm{mt}}$ can be relayed across the organism to trigger a distant $\mathrm{UPR}^{\mathrm{mt}}$ in the intestine, which is coupled to a positive effect on lifespan ${ }^{75}$. Such a systemic propagation of the UPR ${ }^{\mathrm{mt}}$ requires a defined subset of sensory neurons and interneurons as well as several neurotransmitters, including serotonin, that operate in a complex circuitry ${ }^{75,76}$. How these signalling systems connect to each other has not yet been determined. Intriguingly, the UPR ${ }^{\text {tt }}$ caused by pathogen invasion (via hitherto unclear mechanisms) in C. elegans cells is connected to the transactivation of genes coding for antimicrobial peptides through the stress-activated transcription factor ATFS-1 (REF. ${ }^{77}$ ). This observation is reminiscent of the relationship between CHOP (the putative mammalian orthologue of ATFS- 1$)^{78}$ and innate immune responses in mammalian intestine resulting from the activation of $\mathrm{UPR}^{\mathrm{ER}}$ (see above) ${ }^{72}$, suggesting that intracellular responses to stress became connected to organismal homeostasis rather early during evolution.

In mammalian cells, the UPR ${ }^{\mathrm{mt}}$ is part of the socalled integrated stress response; involves ATF4, ATF5, $\mathrm{CHOP}$ and CCAAT/enhancer-binding protein- $\beta$ $(\mathrm{C} / \mathrm{EBP} \beta)^{4}$; and is associated with a metabolic switch to glycolysis that favours mitochondrial repair ${ }^{79}$. Similar to the UPR ${ }^{\mathrm{ER}}$, the UPR ${ }^{\mathrm{mt}}$ is also relayed across the plasma membrane to initiate local and systemic adaptation to stress, a process that largely depends on mitokines ${ }^{4,70}$, including nuclear-encoded FGF21 (which is also secreted as a consequence of the UPR ${ }^{\mathrm{ER}}$ ) and growth differentiation factor 15 (GDF15). FGF21 produced by skeletal muscle cells in response to mitochondrial dysfunction - which occurs in an ATF4-dependent manner - increases lipid catabolism and promotes mitochondrial biogenesis and browning in adipocytes ${ }^{80}$. Along similar lines, secretion of GDF 15 by skeletal muscle cells undergoing the UPR ${ }^{\mathrm{mt}}$ affects systemic metabolism, whereby GDF15 improves insulin sensitivity by enhancing oxidative metabolism and lipid mobilization in the adipose tissue, liver and muscle ${ }^{81}$. Of note, weight loss induced by GDF15 depends on its cognate receptor GDNF family receptor $\alpha$-like (GFRAL) ${ }^{82}$. GFRAL is expressed in the area postrema of the brainstem, an area that participates in the control of satiety and is the only part of the brain outside of the blood-brain barrier ${ }^{82}$. In line with its links to mitochondrial stress, circulating GDF15 has been proposed as a biomarker for mitochondrial dysfunction in ageing and age-related disorders ${ }^{83}$. Moreover, GDF15 levels are increased in obese individuals $^{84}$. However, somewhat paradoxically GDF15 can be used to treat obesity in multiple organisms, including 

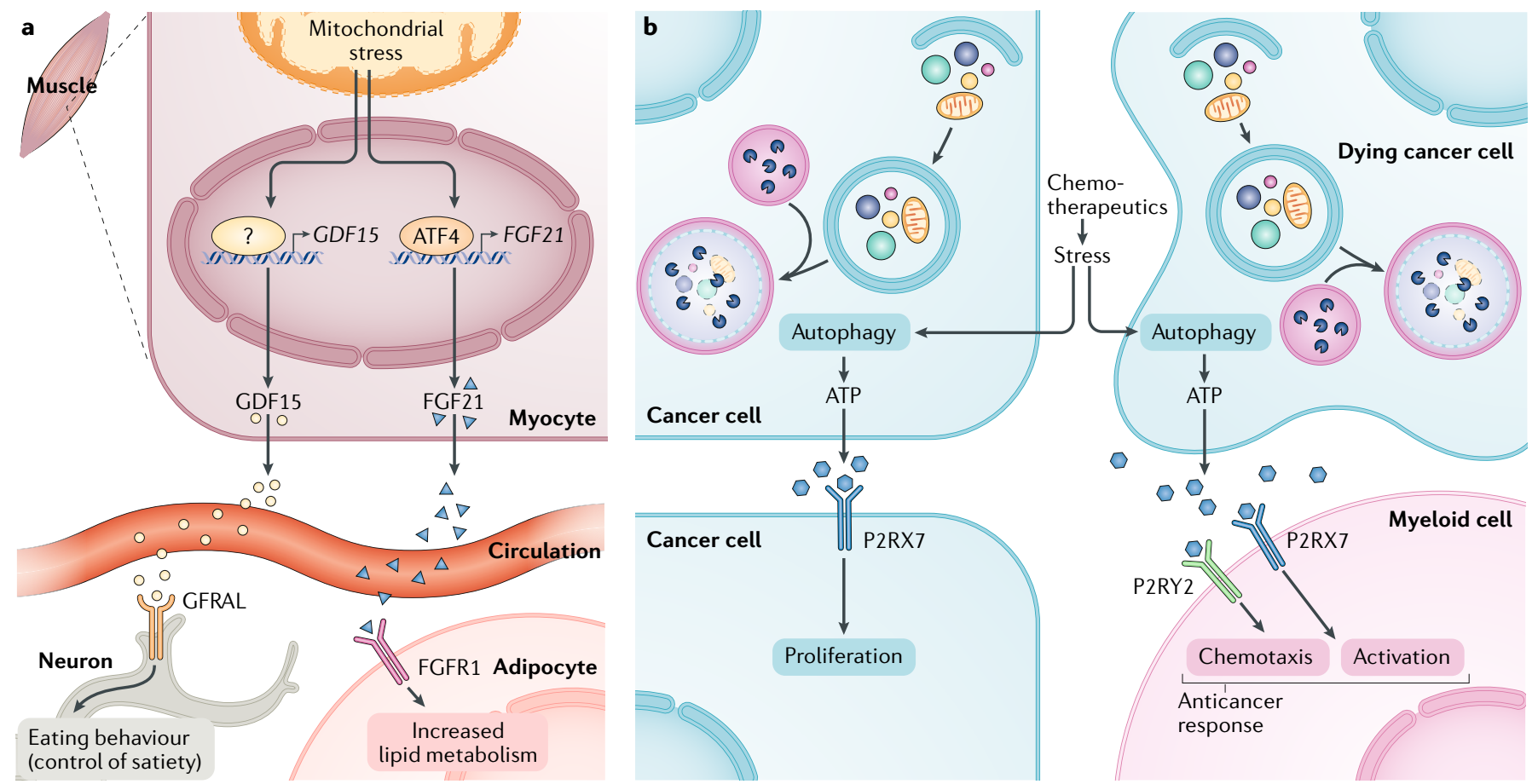

Fig. 3 | Mitochondrial stress responses and autophagy in the regulation of microenvironmental and systemic homeostasis. a | Myocytes experiencing mitochondrial stress mount a transcriptional response that culminates in the release of growth differentiation factor 15 (GDF15) and fibroblast growth factor 21 (FGF21) into the systemic circulation. Upon binding to GDNF family receptor $\alpha$-like (GFRAL) on the surface of neurons of the area postrema of the brainstem, circulating GDF regulates eating behaviour. Blood-borne FGF21 modulates lipid metabolism in the adipose tissue, a process that largely depends on FGF receptor 1 (FGFR1). $\mathbf{b} \mid$ Autophagy-dependent ATP secretion by living cancer cells has been linked to the activation of purinergic receptor P2X7 (P2RX7) in the context of autocrine or paracrine circuitries that stimulate tumour progression. Conversely, the release of ATP by cancer cells that mount unsuccessful autophagic responses to treatment and hence undergo cell death is critical for the recruitment of myeloid cells to the tumour microenvironment, a process that depends on purinergic receptor P2Y2 (P2RY2), and myeloid cell activation, which relies on P2RX7. ATF4, activating transcription factor 4.

AMP-activated protein

kinase

(AMPK). Phylogenetically conserved enzyme expressed by all mammalian cells that has a major role in the regulation of energy metabolism.

Mitochondrial outer membrane permeabilization (MOMP). Loss of integrity of the outer mitochondrial membrane that generally precipitates regulated cell death through apoptosis.

Inflammasome

Supramolecular complex responsible for the caspase 1dependent maturation of IL-1 $\beta$ and IL-1 8 in response to microbial products or other danger signals. rodents and non-human primates ${ }^{85}$. Thus, the elevation of circulating GDF15 documented in the course of obesity may reflect a physiological compensatory mechanism to obesity-associated mitochondrial dysfunction and insulin resistance that is, however, insufficient to affect body mass. Overall, FGF21 and GDF15 exemplify factors that connect intracellular stress (for example, mitochondrial dysfunction) to the regulation of systemic processes (lipid metabolism and eating behaviour, in this specific case) via endocrine mechanisms (FIG. 3a).

Some mitochondrial DNA (mtDNA)-encoded peptides also act as mitokines, although their actual link with the UPR ${ }^{\mathrm{mt}}$ remains to be elucidated. These peptides, which are generally encoded by small, alternative reading frames of the mitochondrial genome, include humanin, multiple small humanin-like peptides (SHLPs) and mitochondrial-derived peptide MOTSc (also known as MTRNR 1$)^{86}$. Humanin not only favours proteostasis and has broad cytoprotective effects coupled to the activation of multiple variants of autophagy $y^{87,88}$ but also may be involved in the regulation of systemic glucose homeostasis $^{89}$. Along similar lines, MOTSc has been attributed a robust role in the preservation of organismal metabolic homeostasis, most likely reflecting its ability to trigger AMP-activated protein kinase (AMPK) activation in the skeletal muscle ${ }^{90}$. Of note, exogenously administered
MOTSc appears to boost the ability of mice to control methicillin-resistant Staphylococcus aureus infection as a consequence of enhanced bactericidal functions by macrophages ${ }^{91}$. Thus, some mitokines may have immunomodulatory effects that participate in the control of systemic homeostasis during infection. SHLP2 also resembles humanin in its cytoprotective effects and its ability to support glucose homeostasis ${ }^{92}$. The biological effects of other SHLPs remain to be explored.

Mitochondrial stressors other than the accumulation of unfolded proteins (for example, respiratory chain poisons) and several other perturbations of cellular homeostasis can culminate in mitochondrial outer membrane permeabilization (MOMP), which is generally an irreversible step in the cascade of events leading to apoptosis ${ }^{10}$. However, MOMP affecting a minority of mitochondria does not necessarily drive RCD but robustly relays intracellular stress to the local and systemic microenvironment ${ }^{93}$. The release of mtDNA into the cytosol following MOMP drives cGAS-STING-dependent type I IFN production ${ }^{94,95}$, as well as inflammasome activation ${ }^{96}$, hence favouring the establishment of local inflammation. This process is limited by the proficient removal of damaged mitochondria by autophagy (mitophagy) ${ }^{97,98}$ and is negatively controlled by caspase 3 (CASP 3$)^{94,95}$. 
$\gamma \delta$ T lymphocytes

T cells expressing a $\gamma \delta$ (rather

than an $\alpha \beta$ ) T cell receptor,

which is associated with a fairly

limited antigenic repertoire but

major histocompatibility

complex-independent

recognition.

Cancer-associated

fibroblasts

Fibroblasts that are found in

the tumour microenvironment

and generally support

malignant cells by nutritional

and immunological

mechanisms.

Ketone bodies

Three related compounds

(acetone, acetoacetic acid and

$\beta$-hydroxybutyric acid) that are

produced during the

metabolism of lipids.

Stellate cells

Hepatic or pancreatic cells that

have a major role in the

maintenance of fibrosis. establishment and

Mitochondrial reactive oxygen species, which are overproduced in response to various stresses, not only favour inflammasome activation and the consequent inflammatory response ${ }^{99}$ but also contribute to the optimal transactivation of vascular endothelial growth factor A (VEGFA) driven by hypoxia, orchestrating microenvironment adaptation to decreased oxygen availability via neovascularization ${ }^{100}$. Finally, cardiolipin, a phospholipid that is normally restricted to mitochondrial membranes, can be presented by CD1D molecules on the cell surface to $\gamma \delta$ T lymphocytes, resulting in their activation ${ }^{101}$. However, it remains unclear whether cardiolipin presentation can occur in the context of intracellular adaptation to stress or only following induction of RCD.

Thus, it is clear that mitochondrial stress is communicated to the local microenvironment and the whole organism by a multitude of mechanisms. Interestingly, the UPR ${ }^{\mathrm{mt}}$ exhibits multiple similarities with its ER counterpart, including the ability to trigger FGF21 secretion in an ATF4-dependent manner. This observation suggests that (at least part of) the adaptive mechanisms triggered by loss of cellular proteostasis originate at highly dynamic platforms that tether subdomains of the ER to mitochondria, which are commonly known as mitochondria-associated ER membranes $(\mathrm{MAMs})^{102}$. Additional work is required to elucidate this possibility.

\section{Box $3 \mid$ Autophagy at a glance}

The word autophagy refers to a group of mechanisms that deliver superfluous or potentially dangerous cytoplasmic entities (be they of endogenous or exogenous origin) to the lysosome for degradation. There are at least three different variants of the process: microautophagy, in which the cargo is directly internalized in small vesicles that form at the surface of the lysosome (vacuole in yeast) or late endosomes; chaperone-mediated autophagy, in which proteins bearing one or multiple KFERQ-like motifs are recognized by heat shock protein family A ( $\mathrm{Hsp} 70)$ member 8 (HSPA8) and translocated across the lysosomal membrane by a specific splicing variant of lysosomal associated membrane protein 2A (LAMP2A); and macroautophagy, in which the materials destined for degradation are progressively sequestered by a dedicated double-membrane organelle (the autophagosome), which eventually fuses with the lysosome to generate a so-called autolysosome ${ }^{7}$. Ultimately, lysosomal hydrolases are activated by lysosomal acidification, leading to degradation of the autophagic cargo and recycling of the autophagic products back to the cytoplasm to feed bioenergetic metabolism or repair pathways.

Macroautophagy, which is the best-characterized variant of autophagy in mammalian cells, can be unselective (degrading various disposable components in support of general bioenergetic homeostasis) or can target a specific substrate as part of a very selective response to stress (for example, permeabilized mitochondria, protein aggregates or invading pathogens ${ }^{188}$. The targeted delivery of a substrate to autophagosomes for degradation is generally ensured by autophagy cargo receptors ${ }^{189}$. Importantly, virtually all eukaryotic cells exhibit constitutive autophagic flux in physiological conditions. This constant autophagic activity is paramount for the preservation of homeostasis as it ensures the removal of the by-products of normal cellular functions and promotes physiological organelle turnover. In addition, autophagic degradation increases in response to a variety of stressful conditions, including (but not limited to) nutritional, hormonal, chemical and physical cues ${ }^{188}$. Although autophagy has long been considered as a form of regulated cell death (RCD), it is now well established that autophagy most often mediates cytoprotective effects, especially in mammals. Accordingly, pharmacological or genetic inhibition of autophagy generally sensitizes mammalian cells to RCD driven by stress ${ }^{10}$. Thus, at odds with other intracellular mechanisms of adaptation to stress, autophagy does not actively drive RCD if cellular homeostasis cannot be recovered, at least in the majority of settings.
Autophagy. Autophagy, in many of its variants (BOX 3), has recently emerged as one of the most central mechanisms of adaptation to stress across the eukaryotic kingdom, representing a promising (yet complex) target for the treatment of multiple human disorders ${ }^{103,104}$. In particular, macroautophagy (hereafter referred to as autophagy) degrades potentially harmful or disposable cytoplasmic entities to support cellular homeostasis in physiological conditions as well as during adaptation to stress ${ }^{6}$. Beyond this phylogenetically conserved cell-autonomous cytoprotective effect, autophagy has a variety of microenvironmental and systemic outcomes, which have been demonstrated by elegant studies in which the deletion of core components of the autophagic machinery in specific cells was shown to affect pathophysiological processes in other cells. However, the interpretation of such studies is complicated by the fact that individual components of the autophagic apparatus may have autophagy-unrelated functions. Thus, only studies in which the individual inactivation of several different core constituents of the autophagic machinery yields a similar phenotype should be interpreted as an indication that the phenomenon under scrutiny is indeed caused by an autophagy defect (as opposed to a defect in an autophagy-related process) ${ }^{7}$.

In some cases, the extracellular effects of autophagy are linked to local or systemic metabolism ${ }^{105}$. For instance, cancer-associated fibroblasts provide lactate and ketone bodies to breast cancer cells in an autophagydependent manner ${ }^{106}$. Similarly, pancreatic stellate cells support the progression of pancreatic adenocarcinoma cells by releasing alanine upon activation of autophagy ${ }^{107}$. In these settings, autophagic responses are likely to be driven by the harsh conditions that generally characterize the tumour microenvironment (for example, low nutrient availability and hypoxia) ${ }^{43}$. Autophagy also supports the secretion of ATP from both living and dying cells, which has major microenvironmental effects. For example, ATP secreted by living melanoma cells in an autophagydependent manner promotes tumour progression via autocrine and paracrine mechanisms ${ }^{108}$. However, in the context of RCD, the release of ATP can drive the recruitment of myeloid cells to apoptotic bodies, which is particularly relevant for cell corpse removal during developmental tissue remodelling ${ }^{109}$ and for anticancer therapy, when the release of ATP by dying cancer cells recruits and activates dendritic cells to initiate tumour-targeting immune responses ${ }^{17,110,111}$ (FIG. 3b). Multiple strategies are currently being tested to pharmacologically target the signalling pathways driven by extracellular ATP, especially in the context of cancer therapy ${ }^{112,113}$. Finally, proficient autophagic responses in the liver, skeletal muscle, adipose tissue and pro-opiomelanocortin neurons prevent pathological weight gain and maintain optimal glucose homeostasis throughout the organism via endocrine pathways that remain to be fully elucidated ${ }^{13,114}$. Thus, the local and systemic effects of autophagy induction on metabolism and immune functions have major implications for human pathophysiology.

Autophagy is also required for a form of nonconventional secretion that involves proteins such as interleukin-1 $\beta$ (IL-1 $\beta$ ) $\left(\right.$ REF. $^{115}$ ) and the diazepam-binding 
Pro-opiomelanocortin neurons

Hypothalamic neurons capable of synthesizing proopiomelanocortin (POMC), the precursor of circulating melanocyte stimulating hormone, adrenocorticotropin hormone and $\beta$-endorphin

Non-conventional secretion Process through which intracellular proteins and other cytoplasmic components are released into the extracellular milieu independently of the endoplasmic reticulum and Golgi apparatus.

Paneth cells

Cells from the intestinal epithelium that contribute to the maintenance of the gastrointestinal barrier.

Crohn's disease

Chronic inflammatory condition of the

gastrointestinal tract

associated with an increased

risk of colorectal cancer.

Exosomes

Cell-derived small vesicles that are present in virtually al mammalian fluids, including blood and urine. inhibitor, acyl-CoA-binding protein (DBI) ${ }^{116}$, as well as other cytoplasmic components ${ }^{117}$. The secretion of mature IL-1 $\beta$ (which is produced by the inflammasome) may involve the uptake of the protein by autophagosomes, most likely between the inner and the outer membrane of the organelle (which explains why the protein is not degraded $)^{118}$. This process relies on heat shock protein HSP90a, which binds two KFERQ-like motifs of IL- $1 \beta$ (REF. ${ }^{118}$ ) and culminates with the fusion between autophagosomes and the plasma membrane ${ }^{119}$, resulting in IL- $1 \beta$ secretion. However, autophagy also limits IL- $1 \beta$ production by multiple mechanisms, including the disposal of damaged mitochondria (which are endogenous sources of inflammasome activators; see above) via mitophagy ${ }^{97,98}$, the degradation of assembled inflammasomes $^{120}$ and the catabolism of immature IL- $1 \beta^{121}$. Thus, although autophagy-dependent secretion supports the establishment of local inflammation, degradative autophagic responses quench it. Accordingly, induction of autophagy by caloric restriction or pharmacological stimuli dampens inflammation along with the reduction of extracellular cytokines including IL-1 $\beta$, IL- 6 and $\mathrm{TNF}^{122}$. In addition, IL-10 can promote degradative autophagy in macrophages, hence limiting IL- $1 \beta$ secretion (which, at least in part, contributes to the antiinflammatory effects of IL-10) ${ }^{123}$. Finally, induction of mitophagy can stimulate the conversion of macrophages towards a pro-inflammatory phenotype ${ }^{124}$. Thus, induction of autophagy may mediate anti-inflammatory or pro-inflammatory effects depending on the context. Of note, protein secretion by autophagy can also operate as a salvage pathway when the conventional secretory pathways fail. For instance, the release of lysozyme (an antibacterial molecule) by Paneth cells infected with Salmonella enterica subsp. enterica serovar Typhimurium relies on autophagy because conventional secretion is blocked by bacterial products ${ }^{125}$. This pathway is compromised by mutations in the autophagy-relevant gene $A T G 16 L 1$ that are associated with an increased risk of Crohn's disease ${ }^{125}$.

Altogether, these examples illustrate how induction of autophagy in one cell may result in the emission of signals that affect other cells locally and systemically. At least in some settings, the paracrine and endocrine effects of autophagy appear to depend on whether autophagic responses are successful (and stressed cells are able to recover homeostasis) or not (and stressed cells succumb to RCD). Most likely, this reflects the differential secretion of other signals (for example, metabolites, cytokines and so forth) that influence the interface between the intracellular and extracellular outputs of autophagy. Identifying the signals that are differentially released from cells undergoing successful versus unsuccessful autophagic responses will provide important insights into non-cell-autonomous roles of autophagy.

\section{Failing adaptation to cellular stress}

Prolonged or severe perturbations of homeostasis that cannot be managed by adaptive mechanisms generally culminate with the proliferative inactivation (cellular senescence) or elimination (via RCD) of stressed cells.
Both of these processes influence the maintenance of organismal homeostasis, both directly and via paracrine and endocrine mechanisms.

Cellular senescence. Cellular senescence is a stress response activated by DNA damage to degrees that are irreparable but insufficient to drive RCD, oncogene signalling or other potential threats to organismal

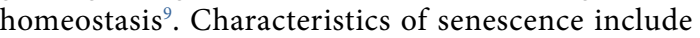
a permanent proliferative arrest linked to the expression of cell cycle inhibitors such as cyclin-dependent kinase inhibitor 2A (CDKN2A) and CDKN1A; a profound metabolic reprogramming often involving autophagy activation; and the secretion of multiple bioactive factors including cytokines, chemokines and extracellular matrix proteins such as IL-1 $\alpha$, IL-6, IL-8, insulin-like growth factor-binding protein 7 (IGFBP7), transforming growth factor- $\beta 1$ (TGF $\beta 1$ ) and oth$\mathrm{ers}^{9}$. The latter property is referred to as senescenceassociated secretory phenotype (SASP) and results from the activation of kinases including MAPK14 and mTOR, epigenetic modifiers such as lysine $N$-methyltransferase $2 \mathrm{~A}$ (encoded by KMT2A) and transcription factors including NF- $\kappa \mathrm{B}, \mathrm{C} / \mathrm{EBP} \beta$, GATA-binding factor 4 (GATA4) and bromodomain-containing protein 4 $(B R D 4)^{126}$. Multiple subtypes of the SASP, differing in the composition of factors that are released, have been characterized ${ }^{127}$. Importantly, a substantial fraction of the SASP-associated secretome is generated by the shedding of membrane-anchored proteins and domains thereof, resulting from the expression of the metalloproteinase ADAM17 (REF. ${ }^{128}$ ). The SASP constitutes a major mechanism whereby cellular senescence is relayed to the local and systemic microenvironment and thus has major pathophysiological and therapeutic relevance.

Cytosolic DNA accumulation resulting in cGASSTING-dependent type I IFN secretion is intimately associated with the establishment of senescence and the SASP (although cGAS-STING activation does not invariably trigger senescence) $)^{23,129}$. Thus, cGAS-deficient or STING-deficient mouse embryonic fibroblasts (MEFs) are resistant to senescence induction and largely deficient in the secretion of IL- 6 and CXC-motif chemokine ligand 10 (CXCL10) ${ }^{129}$. Indeed, STING not only mediates type I IFN production via IRF3 (REF. ${ }^{130}$ ) but also triggers NF- $\kappa \mathrm{B}$ activation via TANK-binding kinase 1 $(\mathrm{TBK} 1)^{131}$. Importantly, type I IFN is sufficient to trigger senescence in both wild-type and cGAS-deficient MEFs, and MEFs lacking IFN $\alpha / \beta$ receptor 1 (IFNAR1) resist oxidative stress-induced senescence. Thus, type I IFN may mediate the autocrine and paracrine propagation of cellular senescence ${ }^{129,132}$. This observation echoes prior reports showing that IL-1 $\alpha$, IL-6, IL-8, IGFB7 and CXCL1 contribute to the autocrine and paracrine maintenance of the senescent phenotype ${ }^{133-135}$. Overall, different components of the SASP can participate in the establishment and perpetuation of senescence as they regulate the local inflammatory milieu. Of note, senescent cells also release increased amounts of exosomes and similar extracellular vesicles that can serve as vessels for diverse cargoes, including proteins, signalling molecules and RNAs ${ }^{136}$ 
Senolytic drugs

Agents that selectively kill senescent cells.

Programmed cell death (PCD). Purely physiological variant of regulated cell death that contributes to post embryonic or embryonic development as well as to the maintenance of adult tissue homeostasis.

\section{Systemic lupus} erythematosus (SLE). Mild to severe autoimmune disease affecting a variety of tissues, including joints, skin, heart and lungs.

LC3-associated phagocytosis Specific variant of the phagocytic process that relies on multiple, but not all, components of the molecular apparatus for autophagy.

Damage-associated molecular patterns (DAMPs). Endogenous molecules that, upon exposure on the plasma membrane or secretion, can be recognized by a pattern recognition receptor and hence participate in the regulation of inflammatory responses.
Senescence is tightly linked to ageing, as senescent cells accumulate with age and have been proposed to support ageing by a variety of mechanisms ${ }^{137}$. Accordingly, systemic elimination of senescent cells upon pharmacological activation of apoptosis (in the context of genetic modifications that enable the specific targeting of senescent cells) or upon administration of senolytic drugs has broad anti-ageing and even rejuvenating effects, at least in preclinical models ${ }^{138-140}$. The current literature suggests a critical pathophysiological role of the SASP in ageing. For example, culture media conditioned by senescent cells increase the osteolytic function of osteoclasts and inhibit bone mineralization by osteoblasts in vitro, in line with the fact that elimination of senescent cells by genetic methods or senolytic drugs improves bone architecture and strength in aged mice ${ }^{141}$. Notably, inhibition of the production of several SASP components was sufficient to counteract bone frailty and age-related bone loss in mice ${ }^{141-143}$. However, it remains to be determined to what extent the anti-ageing effects of senolysis stem from the suppression of SASP. Epistatic experiments testing the elimination of senescent cells in the presence versus the absence of SASP inhibitors might resolve this issue.

Senescence not only is a maladaptive process that accompanies and aggravates ageing but also is a contributor to the maintenance of local and systemic homeostasis. One important role of senescent cells is promoting tissue regeneration, which can occur through secretion of extracellular-matrix-modifying proteases, growthpromoting and stem cell-activating factors and chemoattractants for immune cells ${ }^{144}$ Accordingly, senescent fibroblasts and endothelial cells promote the differentiation of myofibroblasts by secreting platelet-derived growth factor A (PDGFA), and eliminating such senescent cells delays skin wound healing ${ }^{145}$. Furthermore, as mentioned above, senescent cells are recognized and can be removed by NK cells and other lymphocytes. This phenomenon, known as senescence surveillance, reportedly prevents tumorigenesis driven by pre-malignant or malignant hepatocytes ${ }^{20}$. In addition, induction of senescence in hepatocellular carcinoma cells upon restoration of TP53 expression promotes tumour clearance ${ }^{146}$. In this case, p53 is responsible for the secretion of chemokines such as CC-motif chemokine ligand 2 (CCL2) that attract $\mathrm{NK}$ cells and hence enable tumour rejection ${ }^{146}$ (FIG. 4a). Notably, although tumour rejection depends on NKG2D in this setting, induction of senescence by TP53 expression did not promote the expression of NKG2D ligands, suggesting that other mechanisms are at play.

Overall, it is clear that induction of senescence has important consequences for human pathophysiology. In particular, senescence has long been viewed as an acceptable therapeutic goal for cancer therapy, reflecting the permanent proliferative inactivation associated with the senescent state ${ }^{144}$. However, senescence has also been shown to promote tumour progression and resistance to therapy, mostly reflecting the ability of senescent cells to release a number of soluble factors that support cancer cell proliferation and remodel the extracellular matrix ${ }^{144}$. For example, senescent cells can produce exosome-like vesicles containing high levels of ephrin type A receptor 2 (EPHA2), which support tumour progression upon binding to ephrin A1 (EFNA1) on the surface of malignant cells ${ }^{147}$. The extensive intratumoural heterogeneity that characterizes most human neoplasm $\mathrm{s}^{22}$ may also contribute to the different responses mediated by senescent cells in the tumour microenvironment.

Regulated cell death. The links between RCD and systemic homeostasis are multiple and tightly regulated. Programmed cell death (PCD) culminates with efficient removal of cell corpses by tissue-resident macrophages coupled with the delivery of robust anti-inflammatory signals $^{148,149}$. Conversely, RCD occurring in the context of failing adaptation to stress can be linked to the release of cellular content, including various pro-inflammatory signals ${ }^{150}$. The pro-inflammatory effects of RCD can underlie pathogenic maladaptation, as in the case of systemic lupus erythematosus (SLE) ${ }^{150}$. In the course of both PCD and stress-induced RCD, molecules that are exposed on the surface of dying cells or released from them relay RCD to the local (and potentially even systemic) microenvironment. The ultimate outcome of stress-induced RCD largely depends on the precise molecular mechanism of RCD that is engaged, the extent of cell death and the local proficiency of the phagocytic system.

PCD is often immunologically silent. This form of RCD is generally precipitated by apoptotic caspases, notably CASP3 $\left(\mathrm{REF}^{10}\right.$ ), and the activation of CASP3 in dying cells is linked to a variety of anti-inflammatory effects. For instance, CASP3 efficiently prevents cGAS-STING-dependent type I IFN production upon MOMP $^{94,95}$ and is responsible for the production of the mitogenic and immunosuppressive factor prostaglandin $\mathrm{E}_{2}\left(\mathrm{PGE}_{2}\right)^{151,152}$. Moreover, CASP3 underlies the exposure of phosphatidylserine (PtdSer) on the surface of dying cells ${ }^{153,154}$, which delivers robust antiinflammatory signals to macrophages ${ }^{155,156}$ (FIG. 4b). In line with this notion, Jmjd6 ${ }^{-1-}$ mice (which lack the PtdSer receptor) die at birth owing to a robust inflammatory response driven by the accumulation of dead cells in the brain and lungs ${ }^{155,156}$. Similarly, $\mathrm{Rubn}^{-/-}$mice (which are deficient in LC3-associated phagocytosis) spontaneously develop an SLE-like disorder resulting from the accumulation of dead cells ${ }^{157}$. These observations suggest that apoptosis evolved after other RCD modalities as a means to control RCD-driven inflammation, which is of particular importance for embryonic development and adult tissue homeostasis.

In contrast to cells dying by PCD, cells that undergo RCD as a consequence of failing adaptation to stress can be highly immunogenic. These cells secrete a number of damage-associated molecular patterns (DAMPs), which include (but are not limited to) cytokines such as IL-1 $\beta$, type I IFN and CXCL10; metabolic intermediates such as ATP, uric acid and oxidized phospholipids; nucleic acids; nuclear and cytosolic proteins that are normally not secreted, such as high mobility group protein B1 (HMGB1), annexin A1 (ANXA1) and F-actin; and ER chaperones such as CALR and HSP70 (reviewed in REF. ${ }^{17}$ ). IL-1 $\beta$ and type I IFN favour the establishment of local inflammation, whereas CXCL10 operates as a chemoattractant for T lymphocytes ${ }^{158}$. Extracellular ATP has multipronged effects on the local 

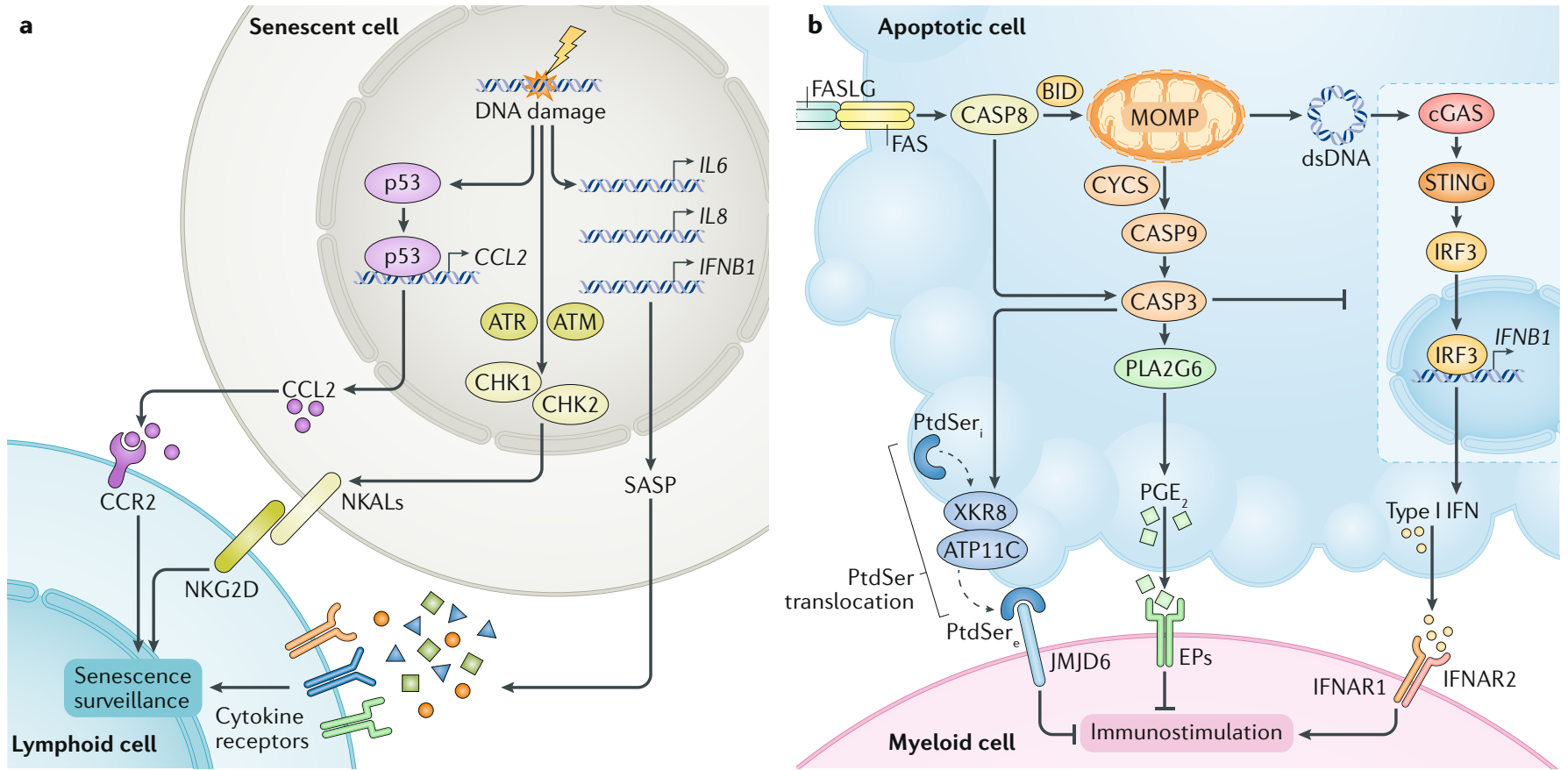

Fig. 4 | Cellular senescence and RCD in the regulation of microenvironmental and systemic homeostasis. a | The establishment of cellular senescence, which often occurs as a consequence of sublethal DNA damage, is associated with a broad transcriptional reprogramming that involves $\mathrm{p} 53$-dependent as well as $\mathrm{p} 53$-independent programmes. Among other consequences, this reprogramming enables the secretion of multiple cytokines and bioactive factors that constitute the so-called 'senescence-associated secretory phenotype' (SASP), including (but not limited to) interferon- $\beta 1$ (IFN $\beta 1$ ), interleukin-6 (IL-6), IL-8 and CC-motif chemokine ligand 2 (CCL2). In conjunction, the activation of ataxia telangiectasia mutated (ATM) and ataxia telangiectasia and Rad3-related protein (ATR) at sites of DNA damage can drive the exposure of natural killer (NK) cell-activating ligands (NKALs) on the cell surface. Collectively, these processes support the eradication of senescent cells by lymphoid cells, a process that is commonly referred to as senescence surveillance. $\mathbf{b}$ | Ligation of FAS cell surface death receptor by FAS ligand (FASLG) initiates a lethal sequence of events that culminates with mitochondrial outer membrane permeabilization (MOMP) and caspase 3 (CASP3) activation, eventually driving apoptotic cell death. CASP3 not only favours the translocation of phosphatidylserine (PtdSer) from the inner ( $\left(\mathrm{tdSSer}_{\mathrm{i}}\right)$ to the outer leaflet

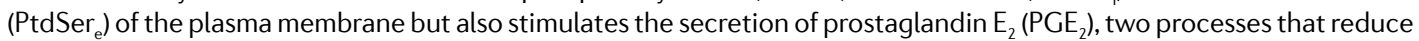
the immunostimulatory potential of dying cells. CASP3 also suppresses the activation of cyclic GMP-AMP synthase (cGAS) signalling in response to the MOMP-dependent release of double-stranded DNA (dsDNA) in the cytosol, thereby preventing the secretion of type I interferon (IFN) and the consequent establishment of local inflammation. ATP11C, ATPase phospholipid transporting 11C; BID, BH3-interacting domain death agonist; CHK, checkpoint kinase; CCR2, CC-motif chemokine receptor 2; CYCS, cytochrome c, somatic; EP, $\mathrm{PGE}_{2}$ receptor EP subtype; IFNAR, IFN $\alpha / \beta$ receptor; IRF3, interferon regulatory factor 3; JMJD6, Jumonji domain-containing 6, arginine demethylase and lysyl hydroxylase; NKG2D, NKG2D type Il integral membrane protein; PLA2G6, phospholipase A2 group VI; RCD, regulated cell death; STING stimulator of interferon genes; XKR8, XK-related 8.

microenvironment, including chemotactic and activatory effects on dendritic cells ${ }^{17}$ and its ability to induce cell death through pyroptosis (which is associated with inflammasome activation and IL- $1 \beta$ production; see also below ${ }^{159}$. Both urate and oxidized phospholipids can trigger inflammasome activation in living myeloid cells ${ }^{160}$, whereas nucleic acids can be recognized

\section{Pyroptosis}

Variant of regulated cell death that is associated with the formation of pores in the plasma membrane by one of multiple gasdermin family members.

RIGI-like receptors (RLRS). Intracellular pattern recognition receptors involved in the recognition of nucleic acids (generally, but not exclusively, of viral origin). by a plethora of pattern recognition receptors, including Toll-like receptor 3 (TLR3), TLR9, cGAS, absent in melanoma 2 (AIM2) and multiple RIGI-like receptors (RLRs), resulting in myeloid cell activation ${ }^{159}$. HGMB1 also mediates immunostimulatory effects upon binding to advanced glycosylation end product-specific receptor (AGER) and TLR4, as does F-actin upon binding to C-type lectin domain family 4 (CLEC4) $)^{17}$. ANXA1 mainly operates to guide the approach of myeloid cells to dying cells via $N$-formyl peptide receptor 1 (FPR1) ${ }^{161}$. Finally, ER chaperones, upon binding to multiple receptors, also act as phagocytic signals ${ }^{17}$. Thus, cells that are eliminated through RCD are capable of releasing various immune stimulating and pro-inflammatory signals. The type of cell death influences - quantitatively, qualitatively and kinetically - the release of these signals.

Pyroptosis generally involves the activation of CASP1, resulting in the proteolytic maturation and release of IL- $1 \beta$ and IL-18 and in the formation of gasdermin D (GSDMD) pores that mediate rapid plasma membrane permeabilization and hence allow for massive DAMP release ${ }^{162}$. In response to specific bacterial components (lipopolysaccharide and lipid A), GSDMD can also be activated by CASP4, CASP 5 or CASP11, resulting in pyroptotic cell death without IL- $1 \beta$ secretion ${ }^{163}$. In addition, in cells treated with certain chemotherapeutics (such as doxorubicin or etoposide), pyroptotic cell death may be induced as a secondary effect of the apoptotic cascade. In this case, pro-apoptotic CASP3 


\section{Necroptosis}

Variant of regulated cell death that involves RIPK3-dependent activation of MLKL, resulting in plasma membrane permeabilization.

Immunogenic cell death (ICD). Functionally defined variant of regulated cell death that is sufficient (in immunocompetent hosts) to establish protective immune responses specific for antigens from dying cells. activates another member of the gasdermin family, GSDME, which drives cell lysis in the absence of IL-1 $\beta$ secretion. This RCD modality may be linked to the side effects of chemotherapy on normal tissues, as cancer cells often express low GSDME levels ${ }^{164}$. Overall, as it is linked to pro-inflammatory cytokine secretion, pyroptosis is inherently immunogenic. In addition, even in the absence of inflammasome activation, formation of gasdermin-based channels during pyroptotic cell death is invariably related to the abundant release of cellular DAMPs into the microenvironment.

Whereas 'accidental' necrosis can mediate immunosuppressive effects by promoting the accumulation of $\mathrm{K}^{+}$ ions in the microenvironment of dying cells and consequent $\mathrm{T}$ cell inhibition ${ }^{165}$, the molecular machinery driving RCD via necroptosis can exert immunostimulatory functions ${ }^{166,167}$. The main transducers of proinflammatory signals during necroptosis are receptor interacting serine/threonine-protein kinase 3 (RIPK3) and mixed lineage kinase domain-like (MLKL), two core components of the necroptotic machinery. Both RIPK3 and MLKL are capable of stimulating inflammasome activation and consequent IL- $1 \beta$, ATP, HGMB1 and type I IFN release, and both are required for the immunogenicity of RCD driven by some chemotherapeutic agents such as anthracyclines and oxaliplatin ${ }^{168-170}$. Moreover, chemical dimerization of RIPK3 favours the activation of an NF- $\kappa \mathrm{B}$-dependent transcriptional programme (involving IL- 6 and CXCL1 secretion) that in some ${ }^{171}$, but not all ${ }^{172}$, models contributes to the immunogenicity of necroptosis. The reasons underlying this discrepancy remain to be elucidated but may relate to context-dependent variables affecting the NF- $\kappa \mathrm{B}$ dependent secretome, such as cell type, necroptosisinducing stimuli and microenvironmental parameters. Additional work is required to clarify this issue.

As mentioned above, apoptotic PCD is usually immunologically silent ${ }^{1}$. However, in response to certain stimuli, including anthracyclines, oxaliplatin and hypofractionated irradiation, apoptosis can mediate robust immunostimulatory effects ${ }^{17,173}$. Such a form of immunogenic cell death (ICD) mechanistically relies on the activation of intracellular stress responses, including (but perhaps not limited to) a partial ER stress response (culminating in the exposure of pro-phagocytic signals see above) ${ }^{63}$, autophagy (resulting in ATP release; see above) ${ }^{110}$ and intracellular nucleic acid sensing (resulting in type I IFN release, see above $)^{25,174}$. Interestingly, apoptotic ICD depends on CASP8 (REF. ${ }^{64}$ ), although it seems that the activation of the pro-inflammatory NF- $\kappa B$ pathway in the course of RCD is robustly inhibited by caspases ${ }^{175}$. Thus, apoptotic ICD stands out as a functionally defined form of RCD that involves caspase activation in the absence of caspase-dependent immunosuppression $^{176}$. Of note, ICD appears to be extremely relevant for cancer therapy as it has been consistently associated with the success of treatment beyond its discontinuation, most likely by participating in the establishment of a clinically useful tumour-targeting immune response ${ }^{161,174,177}$.

Taken together, these observations suggest that RCD induced in response to irreparable damage signals has a major influence on local and systemic inflammatory homeostasis. Such a link stands out as a promising therapeutic target for the management of multiple pathologies in which the unwarranted loss of postmitotic cells is coupled to a pathogenic inflammatory reaction, such as cardiac infarction or neuronal ischaemia ${ }^{178}$, as well as for cancer treatment, in which the activation of tumourtargeting immune responses has become a common therapeutic goal ${ }^{17}$.

\section{Conclusions and perspectives}

In summary, mammalian cells react to potentially harmful perturbations of their microenvironment either by adaptation and damage repair or by irreversible inactivation (via senescence or RCD), and both of these responses are linked to a complex network of signals that connect individual cells to the tissue and the whole organism. Globally, these signals influence a large panel of processes, ranging from local inflammatory reactions to whole-body metabolic homeostasis and feeding behaviour, and their deregulated emission has been linked to multiple pathologies.

It is tempting to speculate that the mechanisms bridging cellular stress responses to the environment (be they local, systemic or interindividual) were established early in the evolution of multicellular life, mostly likely along with the establishment of colonial behaviour. In support of this notion, yeast cells release Acb1 (the orthologue of mammalian DBI) in response to carbon shortage, and extracellular Acb1 reportedly regulates specific colonial activities in a paracrine manner ${ }^{179}$. Of note, many instances of intracellular response to stress in lower eukaryotes (for example, C. elegans) have been directly linked to systemic homeostasis via endocrine mechanisms. Conversely, cellular responses to stress in mammals have been mostly connected to local circuitries impinging on autocrine and/or paracrine signalling or the regulation of inflammatory responses at the microenvironmental level, with a few exceptions ${ }^{13,53}$. We surmise that such an apparent discrepancy in the general organization of organismal stress responses in lower versus higher eukaryotes may largely reflect the different degree of complexity of these organisms.

The paracrine and endocrine pathways elicited by cellular stress have major implications for the treatment of a large panel of human disorders. On the one hand, it has become clear that preventing RCD in ischaemic tissues is generally not achievable. Thus, considerable attention is being devoted to strategies that may modulate the ability of RCD to establish local inflammation rather than the occurrence of RCD itself ${ }^{178}$. On the other hand, it is now established that the activation of tumourtargeting immune responses by anticancer therapy is responsible for the long-term success of treatment. Thus, anticancer therapies are now being aimed at specifically provoking ICD rather than causing a large cytotoxic effect $^{113}$. We suspect that additional mechanisms that relay adaptation to stress from individual cells to the whole organism will be elucidated in the near future, and this will likely change our perception of many other pathophysiological processes. 
1. Fuchs, Y. \& Steller, H. Live to die another way: modes of programmed cell death and the signals emanating from dying cells. Nat. Rev. Mol. Cell Biol. 16, 329-344 (2015)

2. Chang, H. H. Y., Pannunzio, N. R., Adachi, N. \& Lieber, M. R. Non-homologous DNA end joining and alternative pathways to double-strand break repair. Nat. Rev. Mol. Cell Biol. 18, 495-506 (2017).

3. Hetz, C. \& Papa, F. R. The unfolded protein response and cell fate control. Mol. Cell 69, 169-181 (2018).

4. Shpilka, T. \& Haynes, C. M. The mitochondrial UPR mechanisms, physiological functions and implications in ageing. Nat. Rev. Mol. Cell Biol. 19, 109-120 (2018).

5. Suomalainen, A. \& Battersby, B. J. Mitochondrial diseases: the contribution of organelle stress responses to pathology. Nat. Rev. Mol. Cell Biol. 19, 77-92 (2018).

6. Galluzzi, L., Pietrocola, F., Levine, B. \& Kroemer, G Metabolic control of autophagy. Cell 159, 1263-1276 (2014).

7. Galluzzi, L. et al. Molecular definitions of autophagy and related processes. EMBO J. 36, 1811-1836 (2017).

8. Cao, X. Self-regulation and cross-regulation of patternrecognition receptor signalling in health and disease. Nat. Rev. Immunol. 16, 35-50 (2016).

9. Neves, J., Demaria, M., Campisi, J. \& Jasper, H. Of flies, mice, and men: evolutionarily conserved tissue damage responses and aging. Dev. Cell 32, 9-18 (2015).

10. Galluzzi, L. et al. Molecular mechanisms of cell death: recommendations of the Nomenclature Committee on Cell Death 2018. Cell Death Differ. 25, 486-541 (2018).

11. Galluzzi, L., Bravo-San Pedro, J. M., Kepp, O. \& Kroemer, G. Regulated cell death and adaptive stress responses. Cell. Mol. Life Sci. 73, 2405-2410 (2016).

12. Yatim, N., Cullen, S. \& Albert, M. L. Dying cells actively regulate adaptive immune responses. Nat. Rev. Immunol. 17, 262-275 (2017)

13. He, C. et al. Exercise-induced BCL2-regulated autophagy is required for muscle glucose homeostasis. Nature 481, 511-515 (2012).

14. Zhang, Q. et al. Circulating mitochondrial DAMPs cause inflammatory responses to injury. Nature 464 104-107 (2010).

15. Ermolaeva, M. A. \& Schumacher, B. Systemic DNA damage responses: organismal adaptations to genome instability. Trends Genet. 30, 95-102 (2014).

16. Ribezzo, F., Shiloh, Y. \& Schumacher, B. Systemic DNA damage responses in aging and diseases. Semin. Cancer Biol. 37-38, 26-35 (2016).

17. Galluzzi, L., Buque, A., Kepp, O., Zitvogel, L. \& Kroemer, G. Immunogenic cell death in cancer and infectious disease. Nat. Rev. Immunol. 17, 97-111 (2017).

This comprehensive review examines the molecular and cellular mechanism whereby cell death can be perceived as immunogenic by the host and the pathophysiological implication of this process.

18. Gasser, S., Orsulic, S., Brown, E. J. \& Raulet, D. H. The DNA damage pathway regulates innate immune system ligands of the NKG2D receptor. Nature $\mathbf{4 3 6}$ 1186-1190 (2005)

19. Wennerberg, E. et al. Immune recognition of irradiated cancer cells. Immunol. Rev. 280, 220-230 (2017).

20. Kang, T. W. et al. Senescence surveillance of premalignant hepatocytes limits liver cancer development. Nature 479, 547-551 (2011).

This paper provides the first description of senescence surveillance as an immunological mechanism that limits oncogenesis in the liver upon the eradication of senescent hepatocytes.

21. Lopez-Soto, A., Gonzalez, S., Smyth, M. J. \& Galluzzi, L. Control of metastasis by NK cells. Cancer Cell 32 , 135-154 (2017)

22. Galluzzi, L. \& Vitale, I. Oncogene-induced senescence and tumour control in complex biological systems. Cell Death Differ. 25, 1005-1006 (2018).

23. Dou, Z. et al. Cytoplasmic chromatin triggers inflammation in senescence and cancer. Nature 550 402-406 (2017)

24. Mackenzie, K. J. et al. cGAS surveillance of micronuclei links genome instability to innate immunity. Nature 548, 461-465 (2017)

25. Vanpouille-Box, C. et al. DNA exonuclease Trex 1 regulates radiotherapy-induced tumour immunogenicity. Nat. Commun. 8, 15618 (2017)

26. Harding, S. M. et al. Mitotic progression following DNA damage enables pattern recognition within micronuclei. Nature 548, 466-470 (2017). References 23-26 independently demonstrate that multiple conditions associated with DNA damage result in the accumulation of doublestranded DNA in the cytoplasm and consequent release of type I IFN upon CGAS and STING activation

27. Chen, Q., Sun, L. \& Chen, Z. J. Regulation and function of the cGAS-STING pathway of cytosolic DNA sensing. Nat. Immunol. 17, 1142-1149 (2016).

28. Galluzzi, L., Vanpouille-Box, C., Bakhoum, S. F. \& Demaria, S. SnapShot: CGAS-STING signaling. Cell 173, 276-276 (2018)

29. Crow, Y. J. et al. Mutations in genes encoding ribonuclease $\mathrm{H} 2$ subunits cause Aicardi-Goutieres syndrome and mimic congenital viral brain infection. Nat. Genet. 38, 910-916 (2006)

30. Crow, Y. J. et al. Mutations in the gene encoding the 3'-5' DNA exonuclease TREX1 cause AicardiGoutieres syndrome at the AGS1 locus. Nat. Genet. 38, 917-920 (2006)

31. King, K. R. et al. IRF3 and type I interferons fuel a fatal response to myocardial infarction. Nat. Med. 23 , 1481-1487 (2017).

32. Dewan, M. Z. et al. Fractionated but not single-dose radiotherapy induces an immune-mediated abscopal effect when combined with anti-CTLA-4 antibody. Clin. Cancer Res. 15, 5379-5388 (2009)

33. Dewan, M. Z. et al. Synergy of topical toll-like receptor 7 agonist with radiation and low-dose cyclophosphamide in a mouse model of cutaneous breast cancer. Clin. Cancer Res. 18, 6668-6678 (2012).

34. Reijns, M. A. et al. Enzymatic removal of ribonucleotides from DNA is essential for mammalian genome integrity and development. Cell 149, 1008-1022 (2012).

35. Stephens, P. J. et al. Massive genomic rearrangement acquired in a single catastrophic event during cancer development. Cell 144, 27-40 (2011).

This paper characterizes chromothripsis as a single catastrophic event affecting one or a few chromosomes (or fragments thereof) that simultaneously generates tens of hundreds of genomic rearrangements.

36. Vitale, I., Manic, G., Senovilla, L., Kroemer, G. \& Galluzzi, L. Karyotypic aberrations in oncogenesis and cancer therapy. Trends Cancer 1, 124-135 (2015).

37. Valent, A., Penault-Llorca, F., Cayre, A. \& Kroemer, G Change in HER2 (ERBB2) gene status after taxanebased chemotherapy for breast cancer: polyploidization can lead to diagnostic pitfalls with potential impact for clinical management. Cancer Genet. 206, 37-41 (2013).

38. Mitchison, T. J., Pineda, J., Shi, J. \& Florian, S. Is inflammatory micronucleation the key to a successful anti-mitotic cancer drug? Open Biol. 7, 170182 (2017).

39. Xia, T., Konno, H., Ahn, J. \& Barber, G. N. Deregulation of STING signaling in colorectal carcinoma constrains DNA damage responses and correlates with tumorigenesis. Cell Rep. 14, 282-297 (2016).

40. Bartsch, K. et al. Absence of RNase $\mathrm{H} 2$ triggers generation of immunogenic micronuclei removed by autophagy. Hum. Mol. Genet. 26, 3960-3972 (2017).

41. Dou, Z. et al. Autophagy mediates degradation of nuclear lamina. Nature 527, 105-109 (2015).

42. Rello-Varona, S. et al. Autophagic removal of micronuclei. Cell Cycle 11, 170-176 (2012).

43. Rybstein, M. D., Bravo-San Pedro, J. M., Kroemer, G. $\&$ Galluzzi, L. The autophagic network and cancer. Nat. Cell Biol. 20, 243-251 (2018)

44. Dikic, I. \& Elazar, Z. Mechanism and medical implications of mammalian autophagy. Nat. Rev. Mol. Cell Biol. 19, 349-364 (2018)

45. Ermolaeva, M. A. et al. DNA damage in germ cells induces an innate immune response that triggers systemic stress resistance. Nature 501, 416-420 (2013).

46. Peng, Y. et al. Cysteine protease cathepsin B mediates radiation-induced bystander effects. Nature 547 458-462 (2017).

47. Recklies, A. D. Tiltman, K. J., Stoker, T. A \& Poole, A. R. Secretion of proteinases from malignant and nonmalignant human breast tissue. Cancer Res. 40, 550-556 (1980)

48. Shree, T. et al. Macrophages and cathepsin proteases blunt chemotherapeutic response in breast cancer. Genes Dev. 25, 2465-2479 (2011)

49. Bian, B. et al. Cathepsin B promotes colorectal tumorigenesis, cell invasion, and metastasis. Mol. Carcinog. 55, 671-687 (2016).
50. Moon, H. Y. et al. Running-induced systemic cathepsin $B$ secretion is associated with memory function. Cell Metab. 24, 332-340 (2016).

51. Frakes, A. E. \& Dillin, A. The UPR(ER): Sensor and coordinator of organismal homeostasis. Mol. Cell 66 761-771 (2017)

52. Mami, I. et al. A novel extrinsic pathway for the unfolded protein response in the kidney. J. Am. Soc. Nephrol. 27, 2670-2683 (2016).

53. Vecchi, C. et al. ER stress controls iron metabolism through induction of hepcidin. Science 325, 877-880 (2009).

54. Hosomi, S. et al. Intestinal epithelial cell endoplasmic reticulum stress promotes MULT 1 up-regulation and NKG2D-mediated inflammation. J. Exp. Med. 214, 2985-2997 (2017).

55. Miyake, M. et al. Skeletal muscle-specific eukaryotic translation initiation factor 2alpha phosphorylation controls amino acid metabolism and fibroblast growth factor 21-mediated non-cell-autonomous energy metabolism. FASEB J. 30, 798-812 (2016).

56. Bohnert, K. R., McMillan, J. D. \& Kumar, A. Emerging roles of ER stress and unfolded protein response pathways in skeletal muscle health and disease. J. Cell. Physiol. 233, 67-78 (2018)

57. Ozcan, L. et al. Endoplasmic reticulum stress plays a central role in development of leptin resistance. Cell Metab. 9, 35-51 (2009).

58. Williams, K. W. et al. Xbp1s in Pomc neurons connects ER stress with energy balance and glucose homeostasis. Cell Metab. 20, 471-482 (2014).

59. Taylor, R. C. \& Dillin, A. XBP-1 is a cell-nonautonomous regulator of stress resistance and longevity. Cell 153, 1435-1447 (2013).

60. Guan, B. J. et al. A unique ISR program determines cellular responses to chronic stress. Mol. Cell 68, 885-900 e886 (2017).

61. Sundaram, A., Plumb, R., Appathurai, S. \& Mariappan, M. The Sec61 translocon limits IRE 1 alpha signaling during the unfolded protein response. eLife 6, 27187 (2017)

62. Rodvold J J J et al Intercellular transmission of the unfolded protein response promotes survival and drug resistance in cancer cells. Sci. Signal. 10, aah7177 (2017).

The authors of this article demonstrate that the UPR ${ }^{\mathrm{ER}}$ can be transmitted between cancer cells in a process with important implications for tumour progression and response to treatment that they term 'transmissible ER stress'.

63. Bezu, L. et al. elF2alpha phosphorylation is pathognomonic for immunogenic cell death. Cell Death Differ. (2018).

64. Panaretakis, T. et al. Mechanisms of pre-apoptotic calreticulin exposure in immunogenic cell death. EMBO J. 28, 578-590 (2009).

65. Fucikova, J. et al. Calreticulin expression in human non-small cell lung cancers correlates with increased accumulation of antitumor immune cells and favorable prognosis. Cancer Res. 76, 1746-1756 (2016).

66. Fucikova, J. et al. Calreticulin exposure by malignant blasts correlates with robust anticancer immunity and improved clinical outcome in AML patients. Blood 128, 3113-3124 (2016).

67. Osman, R., Tacnet-Delorme, P., Kleman, J. P., Millet, A $\&$ Frachet, P. Calreticulin release at an early stage of death modulates the clearance by macrophages of apoptotic cells. Front. Immunol, 8, 1034 (2017).

68. He, X. Y. et al. Calreticulin fragment 39-272 promotes B16 melanoma malignancy through myeloid-derived suppressor cells in vivo. Front. Immunol. 8, 1306 (2017).

69. De, I., Dogra, N. \& Singh, S. The mitochondrial unfolded protein response: role in cellular homeostasis and disease. Curr. Mol. Med. 17, 587-597 (2017)

70. Moehle, E. A., Shen, K. \& Dillin, A. Mitochondrial proteostasis in the context of cellular and organismal health and aging. J. Biol. Chem. https://doi.org 10.1074/jbc.TM117.000893 (2018)

71. Tian, Y., Merkwirth, C. \& Dillin, A. Mitochondrial UPR a double-edged sword. Trends Cell Biol. 26, 563-565 (2016).

72. Melber, A. \& Haynes, C. M. UPR(mt) regulation and output: a stress response mediated by mitochondrialnuclear communication. Cell Res. 28, 281-295 (2018).

73. Owusu-Ansah, E., Song, W. \& Perrimon, N. Muscle mitohormesis promotes longevity via systemic repression of insulin signaling. Cell 155, 699-712 (2013).

74. Wang, X. \& Auwerx, J. Systems phytohormone responses to mitochondrial proteotoxic stress. Mol. Cell 68, 540-551.e545 (2017). 
75. Shao, L. W., Niu, R. \& Liu, Y. Neuropeptide signals cell non-autonomous mitochondrial unfolded protein response. Cell Res. 26, 1182-1196 (2016).

76. Berendzen, K. M. et al. Neuroendocrine coordination of mitochondrial stress signaling and proteostasis. Cell 166, 1553-1563.e1510 (2016).

77. Pellegrino, M. W. et al. Mitochondrial UPR-regulated innate immunity provides resistance to pathogen infection. Nature 516, 414-417 (2014).

The authors of this paper show that the UPR ${ }^{\mathrm{mt}}$ in $C$. elegans has a major impact on the systemic response to bacterial infection by favouring the secretion of lysozyme and other antimicrobial peptides.

78. Pellegrino, M. W., Nargund, A. M. \& Haynes, C. M Signaling the mitochondrial unfolded protein response. Biochim. Biophys. Acta 1833, 410-416 (2013).

79. Nargund, A. M., Fiorese, C. J., Pellegrino, M. W., Deng, P. \& Haynes, C. M. Mitochondrial and nuclear accumulation of the transcription factor ATFS-1 promotes OXPHOS recovery during the UPR $(\mathrm{mt})$. Mol. Cell 58, 123-133 (2015)

80. Kim, K. H. et al. Autophagy deficiency leads to protection from obesity and insulin resistance by inducing Fgf21 as a mitokine. Nat. Med. 19, 83-92 (2013).

81. Chung, H. K. et al. Growth differentiation factor 15 is a myomitokine governing systemic energy homeostasis. J. Cell Biol. 216, 149-165 (2017).

82. Hsu, J. Y. et al. Non-homeostatic body weight regulation through a brainstem-restricted receptor for GDF15. Nature 550, 255-259 (2017).

83. Fujita, Y., Taniguchi, Y., Shinkai, S., Tanaka, M. \& Ito, M. Secreted growth differentiation factor 15 as a potential biomarker for mitochondrial dysfunctions in aging and age-related disorders. Geriatr. Gerontol. Int 16 (Suppl. 1), 17-29 (2016)

84. Adela, R. \& Banerjee, S. K. GDF-15 as a target and biomarker for diabetes and cardiovascular diseases: a translational prospective. J. Diabetes Res. 2015. 490842 (2015).

85. Xiong, Y. et al. Long-acting MIC-1/GDF15 molecules to treat obesity: evidence from mice to monkeys. Sci. Transl Med. 9, aan8732 (2017)

86. Kim, S. J., Xiao, J., Wan, J., Cohen, P. \& Yen, K. Mitochondrially derived peptides as novel regulators of metabolism. J. Physiol. 595, 6613-6621 (2017)

87. Gong, Z. et al. Humanin is an endogenous activator of chaperone-mediated autophagy. J. Cell Biol. 217, 635-647 (2018)

88. Han, K., Jia, N., Zhong, Y. \& Shang, X. S14G-humanin alleviates insulin resistance and increases autophagy in neurons of APP/PS1 transgenic mouse. J. Cell. Biochem. 119, 3111-3117 (2017).

89. Gidlund, E. K. et al. Humanin skeletal muscle protein levels increase after resistance training in men with impaired glucose metabolism. Physiol. Rep. 4, e13063 (2016)

90. Lee, C. et al. The mitochondrial-derived peptide MOTS-c promotes metabolic homeostasis and reduces obesity and insulin resistance. Cell Metab. 21 443-454 (2015)

91. Zhai, D. et al. MOTS-c peptide increases survival and decreases bacterial load in mice infected with MRSA. Mol. Immunol. 92, 151-160 (2017).

92. Cobb, L. J. et al. Naturally occurring mitochondrialderived peptides are age-dependent regulators of apoptosis, insulin sensitivity, and inflammatory markers. Aging (Albany NY) 8, 796-809 (2016).

93. Galluzzi, L., Kepp, O. \& Kroemer, G. Mitochondria: master regulators of danger signalling. Nat. Rev. Mol. Cell Biol. 13, 780-788 (2012).

94. White, M. J. et al. Apoptotic caspases suppress mtDNA-induced STING-mediated type I IFN production. Cell 159, 1549-1562 (2014).

95. Rongvaux, A. et al. Apoptotic caspases prevent the induction of type I interferons by mitochondrial DNA. Cell 159, 1563-1577 (2014). References 94 and 95 independently demonstrate that MOMP generally allows for the release of mtDNA into the cytosol, resulting in type I IFN production by cGAS and STING unless apoptotic caspases are active.

96. Zhou, R., Yazdi, A. S., Menu, P. \& Tschopp, J. A role for mitochondria in NLRP3 inflammasome activation. Nature 469, 221-225 (2011).

97. Nakahira, K. et al. Autophagy proteins regulate innate immune responses by inhibiting the release of mitochondrial DNA mediated by the NALP3 inflammasome. Nat. Immunol. 12, 222-230 (2011).
98. Zhong, Z. et al. NF-kappaB restricts inflammasome activation via elimination of damaged mitochondria. Cell 164, 896-910 (2016).

References 97 and 98 show that NF-kB-driven mitophagy mediates robust anti-inflammatory effects by disposing of damaged mitochondria before they release endogenous inflammasome activators.

99. Zhou, R., Tardivel, A., Thorens, B., Choi, I. \& Tschopp, J. Thioredoxin-interacting protein links oxidative stress to inflammasome activation. Nat. Immunol. 11, 136-140 (2010).

100. Al-Mehdi, A. B. et al. Perinuclear mitochondrial clustering creates an oxidant-rich nuclear domain required for hypoxia-induced transcription. Sci. Signal 5, ra47 (2012)

101. Dieude, $M$ et al Cardiolipin binds to CD1d and stimulates CD 1d-restricted gammadelta T cells in the normal murine repertoire. J. Immunol. 186 , 4771-4781 (2011).

102. Vance, J. E. MAM (mitochondria-associated membranes) in mammalian cells: lipids and beyond. Biochim. Biophys. Acta 1841, 595-609 (2014).

103. Levy, J. M. M., Towers, C. G. \& Thorburn, A. Targeting autophagy in cancer. Nat. Rev. Cancer 17, 528-542 (2017).

104. Galluzzi, L., Bravo-San Pedro, J. M., Levine, B., Green, D. R. \& Kroemer, G. Pharmacological modulation of autophagy: therapeutic potential and persisting obstacles. Nat. Rev. Drug Discov. 16 487-511 (2017).

105. Martinez-Outschoorn, U. E, Peiris-Pages, M. Pestell, R. G., Sotgia, F. \& Lisanti, M. P. Cancer metabolism: a therapeutic perspective. Nat. Rev. Clin. Oncol. 14, 11-31 (2017).

106. Capparelli, C. et al. Autophagy and senescence in cancer-associated fibroblasts metabolically supports tumor growth and metastasis via glycolysis and ketone production. Cell Cycle 11, 2285-2302 (2012).

107. Sousa, C. M. et al. Pancreatic stellate cells support tumour metabolism through autophagic alanine secretion. Nature 536, 479-483 (2016).

This study is the first demonstration that autophagic responses in pancreatic stellate cells allow for the release of alanine in the tumour microenvironment, where it can be taken up by malignant cells to support tumour progression.

108. Martin, S. et al. An autophagy-driven pathway of ATP secretion supports the aggressive phenotype of BRAF(V600E) inhibitor-resistant metastatic melanoma cells. Autophagy 13, 1512-1527 (2017).

109. Qu, X. et al. Autophagy gene-dependent clearance of apoptotic cells during embryonic development. Cell 128, 931-946 (2007).

110. Michaud, M. et al. Autophagy-dependent anticance immune responses induced by chemotherapeutic agents in mice. Science 334, 1573-1577 (2011). The authors of this study report that the immunogenicity of anthracycline-driven cell death obligatorily relies on the activation of autophagic responses that precede RCD and enable robust ATP release.

111. Elliott, M. R. et al. Nucleotides released by apoptotic cells act as a find-me signal to promote phagocytic clearance. Nature 461, 282-286 (2009).

112. Buque, A. et al. Trial Watch-Small molecules targeting the immunological tumor microenvironment for cancer therapy. Oncoimmunology 5, e1149674 (2016).

113. Galluzzi, L., Bravo-San Pedro, J. M., Demaria, S., Formenti, S. C. \& Kroemer, G. Activating autophagy to potentiate immunogenic chemotherapy and radiation therapy. Nat. Rev. Clin. Oncol. 14 , 247-258 (2017)

114. Martinez-Lopez, N. et al. System-wide benefits of intermeal fasting by autophagy. Cell Metab. 26 856-871 (2017).

115. Dupont, N. et al. Autophagy-based unconventional secretory pathway for extracellular delivery of IL-1 beta. EMBO J. 30, 4701-4711 (2011).

116. Loomis, W. F., Behrens, M. M., Williams, M. E. \& Anjard, C. Pregnenolone sulfate and cortisol induce secretion of acyl-CoA-binding protein and its conversion into endozepines from astrocytes. J. Biol. Chem. 285, 21359-21365 (2010)

117. Claude-Taupin, A., Jia, J., Mudd, M. \& Deretic, V. Autophagy's secret life: secretion instead of degradation. Essays Biochem. 61, 637-647 (2017).

118. Zhang, M., Kenny, S. J., Ge, L., Xu, K. \& Schekman, R. Translocation of interleukin- $1 \beta$ into a vesicle intermediate in autophagy-mediated secretion. eLife 4, 11205 (2015)
119. Kimura, T. et al. Dedicated SNAREs and specialized TRIM cargo receptors mediate secretory autophagy. EMBO J. 36, 42-60 (2017)

120. Shi, C. S. et al. Activation of autophagy by inflammatory signals limits IL- 1 beta production by targeting ubiquitinated inflammasomes for destruction. Nat. Immunol. 13, 255-263 (2012).

121. Harris, J. et al. Autophagy controls IL-1 beta secretion by targeting pro-IL-1 beta for degradation. J. Biol. Chem. 286, 9587-9597 (2011).

122. Eisenberg, T. et al. Cardioprotection and lifespan extension by the natural polyamine spermidine. Nat. Med. 22, 1428-1438 (2016)

123. Ip, W. K. E., Hoshi, N., Shouval, D. S., Snapper, S. \& Medzhitov, R. Anti-inflammatory effect of IL-10 mediated by metabolic reprogramming of macrophages. Science 356, 513-519 (2017). This study is the first demonstration that the anti-inflammatory effects of IL-10 result (at least in part) from the activation of a mitophagic response that limits inflammasome activation in macrophages.

124. Esteban-Martinez, L. et al. Programmed mitophagy is essential for the glycolytic switch during cell differentiation. EMBO J. 36, 1688-1706 (2017).

125. Bel, S. et al. Paneth cells secrete lysozyme via secretory autophagy during bacterial infection of the intestine. Science 357, 1047-1052 (2017).

126. McHugh, D. \& Gil, J. Senescence and aging: causes, consequences, and therapeutic avenues. J. Cell Biol. 217, 65-77 (2018).

127. Hoare, M. et al. NOTCH1 mediates a switch between two distinct secretomes during senescence. Nat. Cell Biol. 18, 979-992 (2016)

128. Morancho, B., Martinez-Barriocanal, A., Villanueva, J. $\&$ Arribas, J. Role of ADAM17 in the non-cell autonomous effects of oncogene-induced senescence. Breast Cancer Res. 17, 106 (2015).

129. Gluck, S. et al. Innate immune sensing of cytosolic chromatin fragments through cGAS promotes senescence. Nat. Cell Biol. 19, 1061-1070 (2017).

130. Ishikawa, H \& Barber, G. N. STING is an endoplasmic reticulum adaptor that facilitates innate immune signalling. Nature 455, 674-678 (2008)

131. Ishikawa, H., Ma, Z. \& Barber, G. N. STING regulates intracellular DNA-mediated, type I interferon-dependent innate immunity. Nature 461, 788-792 (2009).

132. Katlinskaya, Y. V. et al. Suppression of type I interferon signaling overcomes oncogene-induced senescence and mediates melanoma development and progression. Cell Rep. 15, 171-180 (2016).

133. Acosta, J. C. et al. Chemokine signaling via the CXCR2 receptor reinforces senescence. Cell 133, 1006-1018 (2008).

134. Kuilman, T. et al. Oncogene-induced senescence relayed by an interleukin-dependent inflammatory network. Cell 133, 1019-1031 (2008).

135. Wajapeyee, N., Serra, R. W., Zhu, X., Mahalingam, M. $\&$ Green, M. R. Oncogenic BRAF induces senescence and apoptosis through pathways mediated by the secreted protein IGFBP7. Cell 132, 363-374 (2008)

136. Lehmann, B. D. et al. Senescence-associated exosome release from human prostate cancer cells. Cancer Res. 68, 7864-7871 (2008)

137. Lopez-Otin, C., Blasco, M. A., Partridge, L., Serrano, M. \& Kroemer, G. The hallmarks of aging. Cell 153, 1194-1217 (2013).

138. Baker, D. J. et al. Naturally occurring p16(Ink4a)positive cells shorten healthy lifespan. Nature $\mathbf{5 3 0}$, 184-189 (2016)

139. Baker, D. J. et al. Clearance of p16Ink4a-positive senescent cells delays ageing-associated disorders. Nature 479, 232-236 (2011).

140. Baar, M. P. et al. Targeted apoptosis of senescent cells restores tissue homeostasis in response to chemotoxicity and aging. Cell 169, 132-147.e116 (2017).

141. Farr, J. N. et al. Targeting cellular senescence prevents age-related bone loss in mice. Nat. Med. 23 1072-1079 (2017)

142. Xu, M. et al. Targeting senescent cells enhances adipogenesis and metabolic function in old age. Elife 4, e12997 (2015).

143. Xu, M. et al. JAK inhibition alleviates the cellular senescence-associated secretory phenotype and frailty in old age. Proc. Natl Acad. Sci. USA 112, E6301-E6310 (2015).

144. Rodier, F. \& Campisi, J. Four faces of cellular senescence. J. Cell Biol. 192, 547-556 (2011).

145. Demaria, M. et al. An essential role for senescent cells in optimal wound healing through secretion of PDGF-AA. Dev. Cell 31, 722-733 (2014). 
146. Iannello, A., Thompson, T. W., Ardolino, M., Lowe, S. W. \& Raulet, D. H. p53-dependent chemokine production by senescent tumor cells supports NKG2D-dependent tumor elimination by natural killer cells. J. Exp. Med. 210, 2057-2069 (2013).

147. Takasugi, M. et al. Small extracellular vesicles secreted from senescent cells promote cancer cell proliferation through EphA2. Nat. Commun. 8, 15729 (2017).

148. Uderhardt, S. et al. 12/15-lipoxygenase orchestrates the clearance of apoptotic cells and maintains immunologic tolerance. Immunity 36, 834-846 (2012)

149. Roberts, A. W. et al. Tissue-resident macrophages are locally programmed for silent clearance of apoptotic cells. Immunity 47, 913-927 (2017).

150. Mistry, P. \& Kaplan, M. J. Cell death in the pathogenesis of systemic lupus erythematosus and lupus nephritis. Clin. Immunol. 185, 59-73 (2017).

151. Huang, Q. et al. Caspase 3-mediated stimulation of tumor cell repopulation during cancer radiotherapy. Nat. Med. 17, 860-866 (2011).

152. Zelenay, S. et al. Cyclooxygenase-dependent tumor growth through evasion of immunity. Cell 162 , 1257-1270 (2015)

153. Suzuki, J., Denning, D. P., Imanishi, E., Horvitz, H. R. $\&$ Nagata, S. Xk-related protein 8 and CED-8 promote phosphatidylserine exposure in apoptotic cells. Science 341, 403-406 (2013).

154. Segawa, K. et al. Caspase-mediated cleavage of phospholipid flippase for apoptotic phosphatidylserine exposure. Science 344, 1164-1168 (2014).

155. Fadok, V. A. et al. A receptor for phosphatidylserinespecific clearance of apoptotic cells. Nature $\mathbf{4 0 5}$ 85-90 (2000).

156. Li, M. O., Sarkisian, M. R., Mehal, W. Z., Rakic, P. \& Flavell, R. A. Phosphatidylserine receptor is required for clearance of apoptotic cells. Science 302 , 1560-1563 (2003).

References 155 and 156 characterize the receptor for PtdSer and its critical importance for the silent removal of apoptotic cells in the context of PCD.

157. Martinez, J. et al. Noncanonical autophagy inhibits the autoinflammatory, lupus-like response to dying cells. Nature 533, 115-119 (2016). The authors of this study demonstrate that defects in LC3-associated phagocytosis cause deficient removal of dead cells by phagocytosis, culminating in an autoimmune disease similar to SLE, at least in mice.

158. Zitvogel, L., Galluzzi, L., Kepp, O., Smyth, M. J. \& Kroemer, G. Type I interferons in anticancer immunity. Nat. Rev. Immunol. 15, 405-414 (2015).

159. Franz, K. M. \& Kagan, J. C. Innate immune receptors as competitive determinants of cell fate. Mol. Cell 66, 750-760 (2017).

160. Zanoni, I., Tan, Y., Di Gioia, M., Springstead, J. R. \& Kagan, J. C. By capturing inflammatory lipids released from dying cells, the receptor CD14 induces inflammasome-dependent phagocyte hyperactivation. Immunity 47, 697-709 (2017).

161. Vacchelli, E. et al. Chemotherapy-induced antitumor immunity requires formyl peptide receptor 1 . Science 350, 972-978 (2015).

162. Jorgensen, I., Rayamajhi, M. \& Miao, E. A. Programmed cell death as a defence against infection. Nat. Rev. Immunol. 17, 151-164 (2017).

163. Shi, J. et al. Inflammatory caspases are innate immune receptors for intracellular LPS. Nature 514, 187-192 (2014).

164. Wang, Y. et al. Chemotherapy drugs induce pyroptosis through caspase- 3 cleavage of a gasdermin. Nature 547, 99-103 (2017).

This paper provides the first description of a pyroptotic variant of RCD that depends on CASP3 and GSDME, rather than on inflammatory caspases and GDSMD, which might contribute to the side effects of chemotherapy.

165. Eil, R. et al. Ionic immune suppression within the tumour microenvironment limits T cell effector function. Nature 537, 539-543 (2016).

166. Galluzzi, L., Kepp, O., Chan, F. K. \& Kroemer, G. Necroptosis: mechanisms and relevance to disease. Annu. Rev. Pathol. 12, 103-130 (2017).

167. Weinlich, R., Oberst, A., Beere, H. M. \& Green, D. R. Necroptosis in development, inflammation and disease. Nat. Rev. Mol. Cell Biol. 18, 127-136 (2017)

168. Yang, H. et al. Contribution of RIP3 and MLKL to immunogenic cell death signaling in cancer chemotherapy. Oncoimmunology 5 , e1149673 (2016).

169. Conos, S. A. et al. Active MLKL triggers the NLRP3 inflammasome in a cell-intrinsic manner. Proc. Natl Acad. Sci. USA 114, E961-E969 (2017).

170. Vince, J. E. et al. Inhibitor of apoptosis proteins limit RIP3 kinase-dependent interleukin-1 activation. Immunity 36, 215-227 (2012).

171. Yatim, N. et al. RIPK 1 and NF-kappaB signaling in dying cells determines cross-priming of $\mathrm{CD} 8^{+} \mathrm{T}$ cells. Science 350, 328-334 (2015)

172. Aaes, T. L. et al. Vaccination with necroptotic cancer cells induces efficient anti-tumor immunity. Cell Rep. 15, 274-287 (2016) References 171 and 172 demonstrate that RIPK3-driven necroptotic cell death is immunogenic and is accompanied by the activation of an NF-kB-dependent transcriptional response.

173. Kepp, O. et al. Consensus guidelines for the detection of immunogenic cell death. Oncoimmunology 3 , e955691 (2014)

174. Sistigu, A. et al. Cancer cell-autonomous contribution of type I interferon signaling to the efficacy of chemotherapy. Nat. Med. 20, 1301-1309 (2014).

175. Giampazolias, E. et al. Mitochondrial permeabilization engages NF-кB-dependent anti-tumour activity under caspase deficiency. Nat. Cell Biol. 19, 1116-1129 (2017).

176. Galluzzi, L., Lopez-Soto, A., Kumar, S. \& Kroemer, G. Caspases connect cell-death signaling to organismal homeostasis. Immunity $\mathbf{4 4}$ 221-231 (2016)

177. Senovilla, L. et al. An immunosurveillance mechanism controls cancer cell ploidy. Science 337, 1678-1684 (2012).

178. Galluzzi, L. et al. Essential versus accessory aspects of cell death: recommendations of the NCCD 2015 Cell Death Differ. 22, 58-73 (2015).

179. Xu, X., Zhao, Y., Kirkman, E. \& Lin, X. Secreted Acb contributes to the yeast-to-hypha transition in Cryptococcus neoformans. Appl. Environ. Microbiol. 82, 1069-1079 (2015)

180. Vitale, I., Manic, G., De Maria, R., Kroemer, G. \& Galluzzi, L. DNA damage in stem cells. Mol. Cell 66 306-319 (2017)

181. Luo, S., Baumeister, P., Yang, S., Abcouwer, S. F. \& Lee, A. S. Induction of Grp78/BiP by translational block: activation of the Grp78 promoter by ATF4 through and upstream ATF/CRE site independent of the endoplasmic reticulum stress elements. J. Biol. Chem. 278, 37375-37385 (2003).

182. Lee, A. H., Iwakoshi, N. N. \& Glimcher, L. H. XBP-1 regulates a subset of endoplasmic reticulum resident chaperone genes in the unfolded protein response. Mol. Cell. Biol. 23, 7448-7459 (2003).
183. Haze, K., Yoshida, H., Yanagi, H., Yura, T. \& Mori, K. Mammalian transcription factor ATF6 is synthesized as a transmembrane protein and activated by proteolysis in response to endoplasmic reticulum stress. Mol. Biol. Cell 10, 3787-3799 (1999).

184. Johannes, G. \& Sarnow, P. Cap-independent polysomal association of natural mRNAs encoding c-myc, BiP, and eIF4C conferred by internal ribosome entry sites. RNA 4, 1500-1513 (1998).

185. Novoa, I., Zeng, H., Harding, H. P. \& Ron, D. Feedback inhibition of the unfolded protein response by GADD34-mediated dephosphorylation of eIF2alpha. J. Cell Biol. 153, 1011-1022 (2001).

186. Zinszner, $\mathrm{H}$. et al. CHOP is implicated in programmed cell death in response to impaired function of the endoplasmic reticulum. Genes Dev. 12, 982-995 (1998).

187. Galluzzi, L., Bravo-San Pedro, J. M. \& Kroemer, G. Organelle-specific initiation of cell death. Nat. Cell Biol. 16, 728-736 (2014).

188. Sica, V. et al. Organelle-specific initiation of autophagy. Mol. Cell 59, 522-539 (2015).

189. Stolz, A., Ernst, A. \& Dikic, I. Cargo recognition and trafficking in selective autophagy. Nat. Cell Biol. 16 495-501 (2014).

Acknowledgements

The authors apologize to the authors of several high-quality articles dealing with the links between intracellular stress responses and the regulation of organismal homeostasis that were not able to be discussed and cited owing to space limitations. L.G. is supported by a start-up grant from the Department of Radiation Oncology at Weill Cornell Medicine (New York, NY, USA), by industrial grants from Lytix (Oslo, Norway) and Phosplatin (New York, NY, USA), and by donations from Sotio a.s. (Prague, Czech Republic), the Luke Heller TECPR2 Foundation (Boston, MA, USA) and Phosplatin (New York, NY, USA). G.K. is supported by the Ligue contre le Cancer Comitê de Charente-Maritime (Équipe Labellisée); the Agence National de la Recherche (ANR) - Projets Blancs; ANR under the framework of E-Rare-2, the ERA-Net for Research on Rare Diseases; the Association pour la Recherche sur le Cancer (ARC); Cancéropôle lle-de-France; Chancelerie des Universitês de Paris (Legs Poix), the Fondation pour la Recherche Médicale (FRM); a donation by Elior; the European Commission (ArtForce); the European Research Council (ERC); the Fondation Carrefour; the Institut National du Cancer (INCa); INSERM (HTE); the Institut Universitaire de France; the LeDucq Foundation; the LabEx Immuno-Oncology; RHU Torino Lumière; the Seerave Foundation; the SIRIC Stratified Oncology Cell DNA Repair and Tumour Immune Elimination (SOCRATE); and the SIRIC Cancer Research and Personalized Medicine (CARPEM). L.G.'s homepage: http://www.galluzzilab.com. G.K.'s homepage: http://www.kroemerlab.com.

\section{Author contributions}

All authors researched data for the article, wrote the article and edited the manuscript. L.G. and G.K. contributed to discussion of the content before submission.

\section{Competing interests}

The authors declare no competing interests.

Publisher's note

Springer Nature remains neutral with regard to jurisdictional claims in published maps and institutional affiliations.

\section{Reviewer information}

Nature Reviews Molecular Cell Biology thanks C. Hetz, B. Schumacher and the other anonymous reviewer(s) for their contribution to the peer review of this work. 